\title{
Light attenuation in photobioreactors and algal pigmentation under different growth conditions - Model identification and complexity assessment
}

\author{
Wágner, Dorottya Sarolta; Valverde-Pérez, Borja; Plósz, Benedek G.
}

\section{Published in:}

Algal Research

Link to article, DOI:

10.1016/j.algal.2018.08.019

Publication date:

2018

Document Version

Peer reviewed version

Link back to DTU Orbit

Citation (APA):

Wágner, D. S., Valverde-Pérez, B., \& Plósz, B. G. (2018). Light attenuation in photobioreactors and algal pigmentation under different growth conditions - Model identification and complexity assessment. Algal Research, 35, 488-499. https://doi.org/10.1016/j.algal.2018.08.019

\section{General rights}

Copyright and moral rights for the publications made accessible in the public portal are retained by the authors and/or other copyright owners and it is a condition of accessing publications that users recognise and abide by the legal requirements associated with these rights.

- Users may download and print one copy of any publication from the public portal for the purpose of private study or research.

- You may not further distribute the material or use it for any profit-making activity or commercial gain

- You may freely distribute the URL identifying the publication in the public portal 
1 Light attenuation in photobioreactors and algal pigmentation under different

2 growth conditions - model identification and complexity assessment

Dorottya S. Wágnera,b*, Borja Valverde-Pérez ${ }^{\mathrm{a}}$, Benedek Gy. Plósz ${ }^{\mathrm{a}, \mathrm{c}, *}$

$4 \quad{ }^{a}$ Department of Environmental Engineering, Technical University of Denmark, Miljøvej, Building 115, $2800 \mathrm{Kgs}$.

5 Lyngby, Denmark

6

${ }^{c}$ Department of Chemical Engineering, University of Bath, Claverton Down, Bath BA2 7AY, UK

7

*Corresponding authors:dsw@bio.aau.dk; b.g.plosz@bath.ac.uk

8 Abstract

9 Microalgae are photosynthetic organisms, and thus one of the most important factors affecting their 10 growth is light. Effective design and operation of cultivation systems requires mathematically 11 consistent simulation models that can accurately predict light availability and its impact on microalgae growth in photobioreactors (PBR). Three cylindrical column reactors, mimicking typical open pond reactors, with different diameters were used to conduct experiments where the light distribution was monitored inside the reactor. A batch experiment was conducted where the effect of nutrients and light availability on the pigmentation of the microalgae was monitored together with the light distribution. The effect of reactor size and cultivation conditions on the light distribution in PBRs was evaluated. Moreover, we assessed the effect of using different simulation model structures on the model prediction accuracy and uncertainty propagation. Results obtained show that light scattering can have a significant effect on light distribution in reactors with narrow diameter (typical to panel-type PBRs) and under cultivation conditions that promote low

21 pigmentation. The light attenuation coefficient was estimated using the Lambert-Beer equation and it was compared to Schuster's law. The light attenuation was found to be dependent on biomass ${ }^{\mathrm{b}}$ Current address: Department of Chemistry and Bioscience, Aalborg University, Fredrik Bajers Vej 7H, 
concentration and microalgae pigmentation. Using a discretized layer model to describe the light distribution in PBRs resulted in the most accurate prediction of microalgal growth and lowest uncertainty on model predictions.

\section{Keywords}

Green microalgae; Pigments; Light attenuation; Photobioreactor operation; Model identification

\section{Introduction}

Optimizing microalgal cultivation is critical for effective reactor operation. One of the most important factors affecting microalgal growth is light availability [1]. Light is essential for microalgae to conduct photosynthesis and photoautotrophic cultivation is not viable without sufficient light in the reactor [2]. During photosynthesis microalgae convert carbon dioxide and water into carbohydrates and oxygen using light as an energy source [3]. In the light reactions, the light harvesting antenna collects the incoming light (i.e., photons) that is transported to the reaction centres (PSI and PSII) where this energy is converted into chemical energy in the form of $\mathrm{NADPH}_{2}$ and ATP [4]. In the dark reaction or Calvin cycle the produced chemical energy is used to reduce carbon dioxide to phosphoglycerate, which can be further converted to, e.g., carbohydrates [4]. In closed photobioreactors (PBR), the light is more efficiently distributed as a result of optimal reactor designs, e.g., flat-panel [5]. However, in open pond cultivation systems, 90\% of the incoming light intensity is absorbed in the first few centimetres of the culture, resulting in an inefficient distribution of photons [6]. Consequently, effective mixing is required to ensure that microalgal cells are regularly exposed to light [7]. Therefore for proper design of algal cultivation systems, the application of process models that accurately describe light distribution dynamics is essential [8]. Another factor affecting microalgae cultivation in open pond cultivation is the potential contamination by bacteria or protozoa [6]. Open cultivation of microalgae is used especially in used 
water resource recovery systems, where the potential for bacterial contamination is high [9]. The presence of bacteria can further affect the light distribution in PBRs.

There are two major groups of photosynthetic pigments in green algae: chlorophylls (green pigment), absorbing in two spectrum bands (blue (450-475 nm) and red (630-675 nm)), and carotenoids (yellow pigment), absorbing at 400-550 $\mathrm{nm}$. Chlorophylls are the main photonharvesting pigments, whilst carotenoids can serve as protective pigments against high irradiance and reactive oxygen species and improve the light absorbance and the light utilization $[10,11]$. Depending on the culture conditions - mainly nitrogen and light availability - chlorophylls and carotenoids are expressed in different quantities [12-15]. Pigments are also important high value products that can be used as, e.g., food and feed ingredients or cosmetics [11,16-18].

Typically, there are three distinct light regimes prevailing through algal growth. Under light limited conditions, photosynthesis shows linear dependency on light intensity. The maximum photosynthetic rate is reached at saturation light intensity, from where the photosynthetic rate is limited by the dark reactions [2]. Light intensity that is higher than the saturation level causes photoinhibition, whereby the photosynthetic rate declines due to non-photochemical quenching to dissipate the excess energy as heat [19]. Algae exposed to inhibiting light intensities for more than 1 min will be affected by photoinhibition [19]. Due to light dynamics, microalgae have developed acclimation mechanisms to cope with light intensity changes. Regulation occurs in the reaction centres, mainly in PSII, by altering their photon-harvesting capacity or the number of reaction centres [20]. Under light limiting conditions microalgae increase the amount of chlorophyll, i.e. their photon-harvesting capacity. Under high light intensity, chlorophyll levels are reduced to avoid excess energy harvesting [19]. 
Light attenuation in the PBR is affected by the absorption capacity of photosynthetic pigments, the shading effect by cells and light scattering caused by reactor wall and cells [10]. The Lambert-Beer expression accounts for the light absorption in the reactor by the biomass concentration [21] or by the combination of biomass and pigments concentration [22], but does not account for scattering. Schuster's law can be used in cases where the light scattering is considered [21]. When the pigment concentration impact on light distribution is considered, it is necessary to include pigments concentration in the biological model as a state-variable. There are several approaches to model pigment concentration: i) relating the intracellular chlorophyll content to the internal nitrogen quota [22] or to the nitrogen assimilation [23], ii) considering photo-acclimation as the driving force of chlorophyll accumulation [20], or iii) relating the chlorophyll synthesis to inorganic carbon uptake [24]. The dependence of microalgal growth on light intensity can be modelled by following three complexity levels [19]. Type 1 consists of biokinetic models that employ incident or average light intensity, i.e., algal cells are assumed to be exposed to the same light intensity through the entire reactor volume and have the same photosynthetic rate, thus neglecting the effect of photoacclimation and light attenuation (see, e.g.,[25]). Type II models account for light distribution in the culture by applying, e.g., the Lambert-Beer expression (e.g., $[21,26])$ to predict the light intensity at a given reactor depth. Finally, type III models account for culture history in terms of light exposure as cells move around in the system (e.g.,[27]). Light intensity is commonly measured and expressed in the photosynthetically active radiation (PAR) range $(400-700 \mathrm{~nm})($ e.g. $[5,28,29])$.

A microalgal biokinetic process model developed in the framework of activated sludge modelling (ASM-A) was proposed earlier [25], including photoautotrophic and heterotrophic microalgal growth, nitrogen and phosphorus uptake and storage and biomass decay processes. The effect of light intensity on photoautotrophic growth was experimentally assessed and found to be best described by the Steele equation. An average light intensity is used to account for light intensity 
inside the reactor (i.e., Type I model). Moreover, in the paper, the effect of light intensity on heterotrophic growth was assessed. The goal of the ASM-A model is to move towards a consensus based process model for green microalgae. As discussed above, light intensity within PBRs can be accounted for in different ways, which was not evaluated in the original ASM-A biokinetic process model. Thus, to further develop a comprehensive process modelling framework for green microalgae, in this paper, different approaches to predict the effects of light intensity on microalgal growth are assessed.

Hence, the objectives of this study are: (i) to assess the distribution of light intensity in column reactors used for microalgae cultivation with different dimensions, biomass concentrations and pigmentation, receiving light from the top; (ii) to assess the effect of cultivation conditions on the light distribution and the pigment synthesis during batch cultivation; (iii) to identify a process model structure that can describe pigments accumulation and degradation as a function of substrate availability; (iv) to compare different simulation model complexity levels used to predict light intensity in PBRs.

\section{Materials and methods}

\subsection{Microalgae and culture media}

A mixed green microalgal consortium consisting mainly of Chlorella sorokiniana and Scenedesmus $s p$. was used in this study [25]. The mixed culture was cultivated using the MWC+Se synthetic medium [30] by adjusting the nitrogen and phosphorus concentrations as later specified. The consortium was also grown in effluent water from a laboratory-scale enhanced biological phosphorus removal (EBPR) system [31] operated at 16 days of solids retention time (SRT) fed with pre-clarified used water from Lundtofte WWTP (Kgs. Lyngby, Denmark). 


\subsection{Microalgal cultivation in batch reactors}

115 Batch experiments were carried out in an 8-L batch reactor (made out of clear acrylic material, see Fig. S1, Supporting Information (SI)), to assess the effect of nutrients and light availability on the pigments concentration of the microalgae. The cylindrical reactor had a diameter of $140 \mathrm{~mm}$, height of $0.6 \mathrm{~m}$ and working volume of 8-L. Constant aeration with $\mathrm{CO}_{2}$ enriched air $\left(5 \% \mathrm{CO}_{2}\right)$ at a flow rate of $20 \mathrm{~L} / \mathrm{h}$ was used to mix the biomass and to provide $\mathrm{CO}_{2}$. Light was supplied from the top of the reactor with a custom-built lamp, providing $1500 \pm 150 \mu \mathrm{mol}$ photons $\mathrm{m}^{-2} \mathrm{~s}^{-1}$, with a metalhalide light bulb (OSRAM $\odot$, Germany). The reactor wall was covered with a black cloth from the outside to reduce the effect of ambient light on the monitoring of the incoming light intensity. The light sensor (described in section 2.3) was only placed inside the reactor for the course of the light intensity measurements (otherwise it was kept outside of the reactor to not interfere with the light penetration). The inoculum for the batch cultivation was taken from a reactor where the culture was cultivated under light limited conditions due to high biomass concentration (data not reported). 
working volume of 22.5-L. Reactor 2 had a diameter of $140 \mathrm{~mm}$, height of $0.6 \mathrm{~m}$ and working volume of 8-L. Finally, reactor 3 had a diameter of $110 \mathrm{~mm}$, height of $1.2 \mathrm{~m}$ and working volume of 10.5-L. Light was supplied from the top of the reactor from $30 \mathrm{~W}$ fluorescent lamps (Philips, The Netherlands) in case of the tests with synthetic medium. Custom made light source was used during the tests done with used water resources. In order to eliminate ambient light, the reactor walls were covered with a black cloth during the measurements. The incident light intensity measured in each experiment is reported in Table S1, SI. Light intensity distribution in the algae suspension cultivated in synthetic medium was measured for three different concentrations of algal biomass in each reactor. Two tests were conducted using synthetic medium. In the first case microalgae were cultivated under nutrient limited conditions. The light attenuation in the culture was measured on day 1, day 2 and on day 4 of the nutrient limited cultivation. Thus three different concentrations were achieved (Table S1). In the second case microalgae were cultivated in nutrients in excess medium. The culture was grown to reach the highest biomass concentration $(158 \mathrm{mg} / \mathrm{L})$ and the light attenuation was measured. The culture was diluted two times with synthetic medium, to conduct the light attenuation measurements at the lower concentrations as well (at $79 \mathrm{mg} / \mathrm{L}$ and 39.5 $\mathrm{mg} / \mathrm{L})$. More details on the experimental design are reported in the SI, SI-1.

\subsection{Analytical methods}

LI-193 SA Spherical Quantum Sensor (LI-COR, USA) was used to measure the light intensity inside the reactors, connected to a LI-1400 data logger (Fig. S1, SI). The sensor measures within the PAR range. The sensor has a uniform sensitivity to light wavelengths between 400 and $700 \mathrm{~nm}$, which corresponds to light used by algae for photosynthesis. The light intensity sensor was placed in a circular fitting, to ensure that it stayed vertical during the measurement (Fig. S3, SI). It was submerged at the centre of each reactor through the top opening of the reactor and the cable was fitted through a $20 \mathrm{~mm}$ hole in the bottom (Fig. S3, SI). The sensor could be moved up and down 
the reactor column. Light intensity was measured every $2-2.5 \mathrm{~cm}$ over the operational depth of each reactor.

pH was monitored with a pH-electrode Sentix 940 sensor, connected to a MultiLine multi-meter 3430 (WTW, Germany), and dissolved oxygen was monitored using a FDO 925 optical oxygen sensor (WTW, Germany), connected to the same multi-meter.

Total suspended solids (TSS) measurement was carried out using glass fibre filters (Advantec@), USA) with a pore size of $0.6 \mu \mathrm{m}$ based on standard methods [32]. Total nitrogen and phosphorus measurements in the suspension were done using commercial test kits (Hach-Lange@, USA). Ammonium, nitrate, nitrite and phosphate concentrations were measured after sample filtration through $0.2 \mu \mathrm{m}$ syringe filters (Sartorius, Germany) using test kits supplied by Merck@ (USA). The internal cell quota of nitrogen was obtained based on the difference of total nitrogen measured in the algal suspension (algae + medium) and total soluble nitrogen in the filtrate (soluble organic $\mathrm{N}+$ ammonium + nitrite + nitrate). The internal cell quota of phosphorus was calculated by taking the difference of total phosphorus measured in the algal suspension and soluble phosphate measured in the filtrate.

Pigments extraction method was adapted from literature $[11,33]$ and the detailed protocol is reported in the SI, SI-2. The pigments were analysed using ultra high performance liquid chromatography (UHPLC) based on [33]. We targeted chlorophyll $a$ and $b$ as well as some carotenoids (lutein, $\beta$-carotene, violaxanthin) as these were the most common pigments found in Chlorella sp. according to literature [11,16,33,34].

\subsection{Calculations and statistical analysis}

Principle component analysis (PCA) was carried out to assess the relevant correlations between factors that could affect the light attenuation using Matlab (The MathWorks, USA). The variables 
$I=I_{0} * \frac{4 \alpha}{(1+\alpha)^{2} * e^{\delta * X} \operatorname{Alg}^{* Z}-(1-\alpha)^{2} * e^{-\delta * X} \operatorname{Alg}^{* Z}}$ to be able to represent them on the same scale. (Lambert-Beer equation).

The Schuster's law is expressed as [35]:

that were considered in the PCA were chosen to be the internal nitrogen and phosphorus quota, the chlorophyll $\mathrm{a}$ and $\mathrm{b}$ content, the violaxanthin, $\beta$-carotein and lutein concentrations and the biomass concentration. The variables were standardized based on their mean and standard deviation in order

SigmaPlot ${ }^{\circledR}$ was used to fit regression on the experimental data obtained in the three reactors presented in section 2.2. The standard error of the estimate parameter values obtained through the fitting is shown as error bars in the figures in the results section.

The Lambert-Beer expression and the Schuster's law (see, e.g., [21]) were fitted on light distribution curves measured inside the reactor in SigmaPlot ${ }^{\circledR}$ (CA, USA). The two equations were chosen to compare the fitting including light scattering (Schuster's law) and without light scattering

198 where

$$
\alpha=\sqrt{\frac{E_{a}}{E_{a}+E_{s}}} \quad \text { and } \quad \delta=\sqrt{E_{a} *\left(E_{a}+E_{s}\right)}
$$

where $I\left(\mu \mathrm{mol} \mathrm{m} \mathrm{m}^{-1}\right)$ is the light intensity measured at depth $z(\mathrm{~m}), I_{0}\left(\mu \mathrm{mol} \mathrm{m} \mathrm{m}^{-2} \mathrm{~s}^{-1}\right)$ is the incident 201 light intensity, $X_{A l g}\left(\mathrm{~g} \mathrm{~m}^{-3}\right)$ is the biomass concentration, $\mathrm{E}_{\mathrm{a}}$ is the light absorption coefficient and $\mathrm{E}_{\mathrm{s}}$ 202 is the light scattering coefficient.

203 Another approach proposed in this study accounts for light scattering by increasing the measured 204 light path length (depth of the reactor) with a correction factor. An optical path length multiplication 
205 (PLM) factor was determined from the curve fit by fitting the Lambert-Beer equation, modified with the PLM:

where $I\left(\mu \mathrm{mol} \mathrm{m} \mathrm{m}^{-1}\right)$ is the light intensity measured at depth $z(\mathrm{~m}), I_{0}\left(\mu \mathrm{mol} \mathrm{m} \mathrm{m}^{-2} \mathrm{~s}^{-1}\right)$ is the incident light intensity, $k_{a}\left(\mathrm{~m}^{2} \mathrm{~g}^{-1}\right)$ is the attenuation coefficient, $X_{A l g}\left(\mathrm{~g} \mathrm{~m}^{-3}\right)$ is the biomass concentration and PLM (-) is the path length multiplication factor. In this way, the true optical path length caused by scattering was predicted.

\subsection{ASM-A model complexity analysis and model extension}

As discussed earlier, different model complexities are used to account for light intensity in the PBR.

We tested three different assumptions to account for light intensity during model simulations, all of them based on the Lambert-Beer law - see section 3.3. Complexity level 1 (CL-1) assumes that there is a constant average light intensity available in the reactor throughout the simulation. The average light intensity $\left(I_{a v}\right)$, which was set constant over time, was calculated by integration of the Lambert-Beer law as presented in Wágner et al.[25]. CL-2 includes the dynamic calculation of the average light intensity (by integration of the Lambert-Beer law, as in CL-1) for each time-step of the simulation. In this way, light intensity can be updated over time taking into account the impact of biomass concentration. Finally, in CL-3, the culture volume was discretized into $n$ equal layers parallel to each other and orthogonal to the light source, which entered from the top of the reactor. The layer model structure is similar to the model reported by Huesemann et al.[29]. The light intensity is calculated in the middle of each layer using the Lambert-Beer equation. The ASM-A biokinetic model is then solved in each layer for one time-step, whereby different growth rates are expected due to the gradient in light intensity within the PBR. The reactor is modelled as a 
calculated in each layer are then numerically averaged for the entire volume at the end of each timestep and average values were used as initial conditions for the next time-step. The optimal time-step and the number of layers were estimated to be $0.1 \mathrm{~d}$ and 10 layers, respectively, by comparing the root mean square normalized error (RMSNE) of the simulations (Fig. S4, SI). The RMSNE was calculated by comparing the simulation to the experimental data in Batch 1 . The attenuation coefficient present in the Lambert-Beer equation was estimated first based on the TSS concentration (Eq. 3) and then based on the total chlorophyll concentration (Eq. 4) resulting in six assessments in total.

The ASM-A model was extended with the prediction of the chlorophyll content of the microalgae. As previously reported in the literature (e.g.[22]), the chlorophyll content is set proportional to the internal nitrogen quota $\left(\mathrm{X}_{\mathrm{Alg}, \mathrm{N}}\right)$. Chlorophyll is reported to be an easily accessible nitrogen source from the internal nitrogen pool that can be degraded under nitrogen limitation [36]. Thus, it is hypothesized that the chlorophyll that is degraded provides nitrogen to be used inside the cells. We introduced an independent decay term for the chlorophyll content (R7, Table 1), assuming that it is degraded faster than the internal nitrogen content.

\subsection{Model implementation, calibration and evaluation}

The different model structures were implemented in Matlab (The MathWorks, USA) as extensions of the ASM-A simulation model by Wágner et al.[25]. Parameter estimation and model identifiability analysis were carried out based on the Latin-Hypercube-Sampling-based priors for Simplex (LHSS) method [25]. Parameter identifiability is assessed by analysing the posteriori parameter distribution, i.e., parameter $95 \%$ confidence interval and covariance based on 500 
Simplex runs. Values for parameters not estimated in this study were taken from the original ASM-

251 A calibration.

252 The model complexity was compared based on four criteria: (1) model accuracy assessment based 253 on the root mean square normalised error (RMSNE) and Akaike's information criterion (AIC) [37];

254 (2) parameter uncertainty based on the comparison of mean value and 95\% confidence interval; (3) 255 parameter correlation based on [38]; (4) model prediction uncertainty, assessed based on the 95\% 256 confidence bands using average relative interval length (ARIL, based on Dotto et al.[39]) together 257 with the coverage, expressed as ARILC by Ramin et al.[40]. For further details on calculating the 258 above criteria, the reader is referred to the SI, SI-3.

\section{Results and discussion}

\subsection{Estimation of light attenuation under different growth conditions - preliminary} evaluation in short term batch experiments

As light penetrates in a PBR containing a microalgal suspension, there is a decrease in the light intensity with increasing depth (see an example in Fig. 1a). This is due to the light absorption and shading effect by the culture [7]. The Lambert-Beer equation was fitted to light distribution data (see examples in Fig. 1a and 1b) experimentally obtained to estimate the light attenuation coefficient in three PBRs, having different reactor diameters and using three different biomass concentrations. The light attenuation coefficient $\left(k_{a}\right)$ (reported in Table S2, SI) was found to vary as 268 a function of biomass concentrations (Fig. 2a and 2b, Table S3, SI). There was no significant 269 difference in the dependence of $k_{a}$ on biomass concentration between 240 and $140 \mathrm{~mm}$ diameter reactors (Fig. 2a and 2b), whilst the narrowest reactor (110 $\mathrm{mm}$ diameter) showed a different relationship with the biomass concentration. We note that the sensor used to measure the light 272 intensity inside the reactor has a diameter comparable to that of $\mathrm{R} 3(6.1 \mathrm{~cm}$ and $11 \mathrm{~cm}$, 
respectively), which could potentially affect our observations (e.g., increase of light scattering). This factor is assumed to be negligible in influencing measured light intensity data in our study. Additionally, the nutrient availability was found to have significant impact on the predicted light attenuation coefficient (Fig. $2 \mathrm{a}$ and $2 \mathrm{~b}$ ). The bubble size did not significantly (based on standard deviation and student t-test) affect the light attenuation in the PBR (Fig. S5, SI). However, a more dedicated analysis of using different diffusers, mixing-conditions and air-flows should be done to thoroughly evaluate the effect of bubble size on, e.g. light scattering.

\section{$<$ Figure 1 >}

As light penetrates through the culture it can be affected by the scattering from the reactor walls and back-scattering from the microalgal cells [5]. Scattering from the reactor walls can enhance the light intensity as light penetrates through the reactor (see Fig. 1c). Scattering from the reactor walls changes the direction of the light beam as it propagates through the reactor, thereby changing and potentially increasing the true optical path length of light within the algal culture. Therefore, light scattering was quantified by increasing the measured path length with a correction factor (i.e. the optical path length multiplier, PLM), thereby predicting the true optical path length. The LambertBeer equation was fitted on the curves, using $k_{a}$ as estimated in Table S3 (SI) for the nutrient limited and nutrients in excess scenarios. Values of PLM were estimated using the curve fit (Eq. 2, Table S4, SI). The best fit was obtained based on $\mathrm{R}^{2}$. In the case of the narrowest reactor for all tested biomass concentrations, the model predictions can be improved by using the PLM. In case of the wider reactors PLM was only needed for the nutrient limited scenario (Table S4, SI). Under nutrient limited condition the pigment composition changes in the culture, which can result in decreased light absorption by the biomass (Fig. 2a and 2b) compared to nutrients in excess cultivation. Due to the lower light absorption, light scattering can be enhanced by the reactor wall, and thus the use of PLM can improve model prediction. Additionally, Pandey et al.[41] found that 
the wall reflection of light in the reactor has higher impact at lower biomass concentration than in high concentration. Our results (Fig. 2c and 2d) suggest higher attenuation coefficients at low biomass concentrations. Observations made using the PLM were confirmed by fitting Schuster's law on the light distribution curves (Table S5, SI). In case scattering becomes insignificant, the parameter $E_{\mathrm{s}}$ in Schuster's law approaches 0, and thus the expression becomes identical to the Lambert-Beer law. Comparing the attenuation coefficient $\left(\mathrm{k}_{\mathrm{a}}\right)$ estimated using the Lambert-Beer law (Table S2, SI) and $\mathrm{E}_{\mathrm{a}}$ estimated using the Schuster's law (Table S5, SI), indicates that when $\mathrm{E}_{\mathrm{s}}$ is $0, \mathrm{E}_{\mathrm{a}}$ and $\mathrm{k}_{\mathrm{a}}$ are equal or not significantly different. This was found to be the case for $\mathrm{R} 1$ and 2 under in-excess nutrients concentrations, whilst under nutrient limited conditions the Schuster's law gave better fit. In case of R3 (narrowest diameter) a better fit was obtained by applying Schuster's law compared to that of Lambert-Beer under both nutrient limited and in-excess conditions. Thus, modelling the effect of scattering by implementing Schuster's law [21] or the PLM approach is needed to accurately predict light attenuation in reactors with narrow diameter, e.g. flat-plate PBR. However, the prediction by the Lambert-Beer equation, i.e. without accounting for scattering, is sufficient in reactors intended to be used at high biomass concentrations, typically the case in PBRs.

Based on the correlation between the attenuation coefficient and the TSS concentration an exponential relation was obtained, and used to approximate data points (Fig. 2c):

$k_{a}=a * e^{-b * X_{A l g}}$

Eq. 3

where $a\left(\mathrm{~m}^{2} \mathrm{~g}^{-1}\right)$ and $b\left(\mathrm{~m}^{3} \mathrm{~g}^{-1}\right)$ are the correlation parameters estimated and $X_{A l g}\left(\mathrm{~g} \mathrm{~m}^{-3}\right)$ is the biomass concentration.

The light distribution in a PBR also depends on the cultivation conditions, i.e. nutrient availability and culture medium, which can affect microalgal physiology (e.g., pigments content and composition). Under nutrient limited cultivation the estimated attenuation coefficient values are 
significantly higher than for parameters estimated under nutrients in-excess cultivation (Fig. 2c), suggesting that algae absorb less light when algae are cultivated under nutrient limited conditions. As a result of different cultivation conditions, algae change their pigmentation (see Fig. S6, SI). Under nitrogen limitation, chlorophylls are considered to be the first nitrogen pools inside the algae accessed by the cells [14], and thus the chlorophyll content of the algae is expected to decrease together with nutrient availability. Moreover, in more diluted cultures, the light intensity that the algae is exposed to is comparably high, thereby promoting the production of carotenoids serving as photo-protective pigments by capturing energy on characteristic wavelengths [42]. This effect can alter the light absorption of the microalgal cells and thus the light attenuation in the reactor.

\section{$<$ Figure2>}

The composition of cultivation medium can also affect the light distribution in PBR, e.g., treated wastewater contains chromophores and particulate matter that can interfere with light attenuation in PBRs. We assessed the effect of such chromophores on light attenuation in PBR using treated water derived from a laboratory scale EBPR (Fig. 1c and Fig. 2c). We found that using treated used water as cultivation medium resulted in comparably high absorbance (i.e., lower $k_{a}$ ) than using clear and colourless synthetic medium (Fig. 2c). Moreover, we assessed the effect of increasing bacterial biomass concentration on light attenuation. Increasing bacterial biomass concentration can further increase light absorption in the reactor (Fig. 2d). Thus, experiments designed for the estimation of the attenuation coefficient should be carried out using the cultivation medium relevant for the system. This effect is crucial to model combined bacterial-algal cultivation systems where the bacterial biomass concentration can vary (e.g. $[43,44])$. 


\subsection{Effect of cultivation condition on pigments synthesis and light attenuation - evaluation} under dynamic conditions

A 16-day batch experiment was run (Batch 1), where after 3 days the nutrients were depleted from the medium, whilst the biomass concentration kept increasing until day 6 (Fig. 3a and 3b). The chlorophyll $a$ and $b$ concentration inside the biomass decreased from the beginning of the experiment, reaching a plateau after 4 days (Fig. 3c). A slight increase in the chlorophyll $a$ (the primary chlorophyll type) content of the microalgae can also be observed by the end of the cultivation (Fig. 3c). Among the measured carotenoids, lutein was present in the highest concentration (Fig. 3d).

\section{$<$ Figure3>}

As opposed to the observed trends in chlorophyll depletion, carotenoids were accumulated in the first 2 days and then depleted until the end of the cultivation period, possibly due to the increase of biomass concentration, which results in reduced light intensity inside the reactor (Fig. 4a). When microalgae are exposed to high light intensities, the chlorophyll production is suppressed and carotenoids are synthesized due to photo-acclimation processes against high light intensity $[11,15,20,21,42,45,46]$. In the beginning of the cultivation the sudden increase of light intensity (average light intensity was $215 \mu \mathrm{mol} \mathrm{m} \mathrm{m}^{-2} \mathrm{~s}^{-1}$ in the start of the cultivation after the inoculum was acclimated to low light intensity) could potentially result in photo-inhibition as suggested by, e.g., García-Camacho et al.[20] or Vaquero et al.[42]. Moreover, Adesanya et al.[24] report the decrease of chlorophyll in Chlorella vulgaris instantaneously after the start of batch cultivation due to nitrogen limitation in the culture with initial nitrogen concentration similar to our case. Ferreira et al.[36] report that microalgae increase their chlorophyll content under low light intensity to harvest light more efficiently, which can be observed in our experiment at the end of the cultivation period. Furthermore, photo-protective pigments such as carotenoids can be used by microalgae to reduce 
the negative effects of elevated light intensity. Carotenoids can dissipate excess light through nonphotochemical processes, and as antioxidants they can reduce the effect of reactive oxygen species $[13,42]$. Thus the increase in carotenoids content in the beginning of the process is likely due to the elevated light intensities. Moreover, the increase in the carotenoid concentration in the end of the experiment (when chlorophyll content increases as well) can be related to the widening of the light absorption spectrum, whereby carotenoids enhance the light harvesting capacity to enhance the photosynthetic activity [36,42].

In the beginning of the second batch experiment (Batch 2), to assess the change in pigments concentration under changing nutrient availability, nutrients were spiked to the starved culture. There is an increase in chlorophyll $a$ and $b$ concentration during the first 2 days (Fig. 3e). This is possibly due to the available nitrogen in the medium that promotes the synthesis of chlorophyll to enhance photosynthesis [21,36]. 2 days after the bulk nitrogen source is depleted, there is a decrease of the chlorophyll $a$ and $b$ concentration. As previously stated, chlorophyll is reported to be an easily degradable nitrogen source for microalgae [36] and under nitrogen starvation chlorophyll is degraded to support growth [14]. Lutein concentration increases slightly in Batch 2 (Fig. 3f). In this case it is unlikely that lutein serves as a photo-protective pigment, as the average light intensity is similar to the one estimated in the end of the cultivation in Batch 1 (Fig. 4a). Likely, lutein serves to widen the light absorption spectrum, to promote effective photosynthesis [36]. Results are subject to the pigment extraction protocol which were demonstrated to be inefficient for lutein extraction [47].

\subsection{Modelling of the effect of chlorophyll on light attenuation}

The total chlorophyll concentration was expressed as nitrogen based on the nitrogen content of chlorophyll in the molecular formula (chlorophyll $a$ : $\mathrm{C}_{55} \mathrm{H}_{72} \mathrm{O}_{5} \mathrm{~N}_{4} \mathrm{Mg}$ and chlorophyll $b$ : 
$\mathrm{C}_{55} \mathrm{H}_{70} \mathrm{O}_{6} \mathrm{~N}_{4} \mathrm{Mg}$; [48]). We found a linear correlation with the nitrogen quota of the microalgae and the total chlorophyll content (Fig. 5), as also suggested by Bernard[22]. Moreover, Ikaran et al.[49] found similar trends between the stored protein and chlorophyll content of microalgae during batch cultivation, where protein is suggested to be part of the nitrogen quota [14]. However, the maximum nitrogen content present as chlorophyll in the total nitrogen quota was about $2 \%$ in our study and thus it forms an insignificant fraction of nitrogen storage. This is in agreement with Geider and La Roche [48], who reported that $0.2-3 \%$ of the intracellular nitrogen is associated with chlorophyll.

\section{$<$ Figure 5>}

The $k_{a}$ was estimated inside the reactor during the course of the 8 - $\mathrm{L}$ batch experiments using the Lambert-Beer expression. Similar to the previous results presented in section 3.1, values of $k_{a}$ change as function of the TSS concentration (Fig. S7, SI), which can be described using an exponential relation (Table S6, SI). Thus, to effectively predict the light distribution in the PBR, the value of $k_{a}$ cannot be expressed as a constant value, but as a variable updated during the cultivation period (Fig. 4b). We calculated the effective attenuation coefficient that is the product of the attenuation coefficient $\left(\mathrm{k}_{\mathrm{a}}\right)$ and the biomass concentration $\left(\mathrm{X}_{\mathrm{Alg}}\right)$, to decouple the effect of biomass concentration on the light attenuation. This value increases (Fig. 4c) during the cultivation period. As the biomass concentration increases and thus the light intensity inside the reactor decreases the effective attenuation coefficient increases, as can be seen in Eq. 2.

Results obtained in a PCA analysis (Fig. 6a) - whereby the smaller the angles between vectors the stronger correlation is [50] - suggest $k_{a}$ to be the most dependent on the chlorophyll $a$ and $b$ content and the internal nitrogen quota and not dependent on the carotenoids, whereas it is negatively correlated with the biomass concentration. Consequently, the $k_{a}$ expressed as a function of total chlorophyll concentration is proposed. We found different trends between the attenuation and the 
total chlorophyll concentration than in the case of TSS (Fig. 6b). The correlation between the cellular pigment content-specific attenuation coefficient $\left(k_{a, p}\right)$ and the chlorophyll concentration is assessed based on analysing different algebraic expressions (in SigmaPlot ${ }^{\circledR}$ ) and it is identified (based on $\mathrm{R}^{2}$ ) as:

$k_{a, p}=\frac{d}{X_{C h l}}-c$

Eq. 4

where $k_{a, p}\left(\mathrm{~m}^{2} \mathrm{~g}^{-1} \mathrm{Chl}\right)$ is the attenuation coefficient specific for pigments, $c\left(\mathrm{~m}^{2} \mathrm{~g}^{-1} \mathrm{Chl}\right)$ and $d\left(\mathrm{~m}^{-1}\right)$ are the regression parameters estimated and $X_{C h l}\left(\mathrm{~g} \mathrm{Chl} \mathrm{m}^{-3}\right)$ is the total chlorophyll concentration that cannot equal zero. Above approximately $4 \mathrm{mg} \mathrm{Chl} / \mathrm{L}, k_{a, p}$ becomes independent of the chlorophyll content (Fig. 6b). The correlation between $k_{a, p}$ and the pigments concentration (based on Eq. 4) is shown in Table S6, SI.

\section{<Figure 6>}

The cellular chlorophyll content can be modelled as a function of the internal nitrogen quota and by introducing a specific chlorophyll decay process rate (R7, Table 1). The specific chlorophyll decay rate coefficient $\left(b_{X C h l}\right)$ was estimated using measured data obtained in Batch 1 . A value of $b_{X C h l}=0.45 \pm 0.043 \mathrm{~d}^{-1}$ was estimated using the LHSS method. The fraction of chlorophyll-nitrogen $\left(f X N_{C h l}\right)$ to the total cellular nitrogen quota was estimated from the slope of Fig. 5 , i.e. $f X N_{C h l}=$ $0.026 \mathrm{gN}-\mathrm{Chl} / \mathrm{gN}$. The chlorophyll concentration can effectively be predicted using the extended ASM-A simulation model (Fig. 7). The variability of $b_{X C h l}$ was assessed using the Janus coefficient $(J)$ by comparing RMSNE values obtained with Batch 1 (used for model calibration) and Batch 2 (used for model validation). $J \sim 1$, thus $b_{X C h l}$ estimate derived from Batch 1 can be used to achieve accurate model prediction in Batch 2 (Fig. S8, SI). In the following section (3.3) we evaluate the difference of calculating $\mathrm{k}_{\mathrm{a}}$ as a function of TSS and chlorophyll content on the model simulations (in all complexity levels CL1-CL3). 


\subsection{Simulation model complexity evaluation}

Three different model structures to predict the impact of light on algal growth were compared, together with different expressions for the light attenuation coefficient $\left(\mathrm{k}_{\mathrm{a}}\right.$ or $\left.\mathrm{k}_{\mathrm{a}, \mathrm{p}}\right)$, using four selection criteria (see section 2.6). Model accuracy assessment was based on RMSNE and AIC calculations (Table S7, SI). Based on these two criteria, the accuracy of the predicted biomass concentration $\left(X_{A l g}\right)$ improved by using a model structure with higher complexity, i.e. the layermodel, regardless of the constitutive equation used to calculate the attenuation coefficient. The sum of RMSNE calculations suggest, as opposed to the AIC results, that there is a worse overall fit with using the layer model. This discrepancy is due to that normalized objective functions, e.g. RMSNE used in this study, result larger values when experimental data are low (e.g., values below 1) [51]. In case of the AIC calculation there is no normalization included (Eq. S2, SI). We hypothesise that, using an average and constant light intensity value might result in the inaccurate prediction of the measurement data in both cases. Under high biomass concentrations the simulation model tends to over-predict the experimental data (Fig. S9 and S10, SI). Implementing the time-variable average light intensity function reduces this over-prediction (Fig. S11 and S12, SI). Finally, using a onedimensional model structure improves the goodness of fit predominantly for the prediction of the biomass concentration (Fig. S13 and S14, SI and Table S7, SI), as a result of the more realistic prediction of light availability for algal growth in the PBR.

The parameter uncertainty was assessed based on the comparison of the mean value and $95 \%$ confidence interval of the parameter subset estimated using the different simulation model complexity levels and attenuation coefficients (Fig. 8, Table S8, SI,). The different model structures do not significantly influence the parameter estimates across the scenarios. The mean estimate for 
the maximum specific growth rate using the layer model structure is similar to the maximum specific growth rate estimated for Chlorella sorokiniana [52], which was estimated in a flat-plate PBR, where no light limitation occurs.

\section{<Figure 8>}

The parameter correlation was compared using the LHSS method. The posteriori parameter distributions were presented as histograms (Table S9-S14, SI). The histograms are narrow and the 95\% confidence interval is low (below 40\%) in case of CL-2 and CL-3. However, in case of CL-1, the $95 \%$ confidence interval is higher than $40 \%$ in case of $\mu_{A, \max }$ and $k_{N O, A l g}$, due to the simplifying assumption of using average light intensity. The covariance matrices show that most of the parameters are identifiable (covariance is below 0.5) in case of CL-2 and CL-3, thus the reduction of uncertainty with more complex model structures might improve parameter identifiability. Interestingly, $\mu_{A, \max }$ and $k_{N O, A l g}$ show correlation in all cases that can be due to the challenges of calibrating $k_{N O, A l g}$, as discussed in Wágner et al.[25].

The model prediction uncertainty was assessed based on the $95 \%$ confidence bands using ARIL divided by the percentage coverage, expressed as ARILC (based on Ramin et al.[40]). The simulation model performance is improved with increasing model structural complexity. The width of the uncertainty bands is reduced as model complexity increases (Fig. 9; Table S7, SI; Fig. S15S19, SI). This is due to the reduced parameter uncertainty, based on 95\% confidence interval (Fig. 8) when using a more complex model to predict light impact on algal growth. However, in the case of the internal nitrogen cell quota there is a significant number of data points outside of the prediction band for both variable light intensity and layer model cases, mainly due to the decrease of the wideness of the prediction. 
479 Using the average light intensity to account for light (CL-1) gave the least accurate predictions. This 480 scenario is furthest from reality as we assume that the light intensity is the same throughout the 481 cultivation, which is not true, because among others, the biomass concentration increases and thus 482 light intensity decreases. Using the variable average light intensity (CL-2) as a measure of 483 modelling light inside the reactor gives comparably more accurate model predictions. This scenario 484 is also closer to reality, as we account with the effect of the change in biomass on light intensity in 485 the reactor. Using a model with discretized layers (CL-3) to predict the light distribution in PBRs 486 resulted in the most accurate prediction of the microalgal growth as well as the reduction of the 487 uncertainty of the overall model output. However, the computational time significantly increases 488 (although the optimal layer number and time-step was optimised in Fig. S4.) in case of using the 489 layer model (up to 100 fold increase compared to CL-1) which can considerably increase the time 490 and computational power needed. CL-2 and CL-3 performed similarly apart from the prediction of 491 biomass concentration (a critical variable in microalgae cultivation). Thus, we conclude that using 492 CL-3 can improve the prediction accuracy especially in case of biomass concentration. Therefore, 493 the modeler should choose between CL-3 and CL-2 depending on the system to be modeled and the 494 accuracy required to predict, e.g., biomass productivity.

\section{<Figure 9>}

\section{4. Conclusions}

497 In this study, we developed a consistent simulation model extension to the ASM-A framework to 498 accurately predict light attenuation and distribution in PBRs using cylindrical PBRs with different diameters and under different cultivation conditions. 
Three different simulation model structures were compared to predict light intensity inside the PBR. Light scattering had an effect on light distribution in reactors with narrow diameter or under cultivation conditions that promote low biomass concentrations and decreased pigmentation. This is important e.g. when biomass is grown for lipid accumulation in PBR under nutrient limitation and one must be careful to account with possible scattering.

Nitrogen limited conditions resulted in the decrease of chlorophyll content, whilst elevated light intensity promoted the synthesis of carotenoids. In the new model, the light attenuation coefficient is predicted as a function of the pigmentation - calculated as total chlorophyll content of microalgae, thus defining it as a dynamic variable. Algal chlorophyll content is predicted by the model as a function of the internal nitrogen quota and the pigment decay process rate.

We propose a consistent simulation model structure using a one-dimensional discretization (layers) to predict the light distribution in PBRs. As a result, more accurate prediction of the microalgal growth as well as the reduction of the uncertainty of the overall model output is obtained. This comes at a cost of increased computational time.

The ASM-A simulation model shows high predictive accuracy with the dynamic laboratory-scale systems. High variability of nutrient loading is typically the case in used water resource recovery systems. Under such conditions, it is also important to consider the effect of the cultivation medium, which is now also accounted for by the developed simulation model.

The significant outcomes of the paper help to better understand and predict the effects of cultivation conditions on light attenuation in PBRs. For practitioners, investigating other cultures, the implementation of the simulation model developed - using rigorous experimental, statistical and computational approaches (used in our previous study and this study) - is straightforward. 


\section{Acknowledgements}

523

524 525

Dorottya S. Wágner thanks the European Commission (E4WATER Project, FP7-NMP-2011.3.4-1 grant agreement 280756) for the financial support. Borja Valverde-Pérez thanks the Integrated Water Technology (InWaTech) project (http://www.inwatech.org) for the financial support. The authors thank Mariann Sæbø and Michael Steidl for conducting some of the experiments and Dr. Arnaud Dechesne for the discussion on the PCA analysis.

\section{Author contribution declaration}

DSW, BGP and BVP contributed to the design of the experiments. Experiments were carried out by DSW, supported by BVP. The analysis and interpretation of the data was carried out by DSW, supported by BGP and BVP. DSW drafted the manuscript; all authors contributed to its revision and completion, and approved the final submission.

\section{Conflict of interest statement}

The authors declare that there are no known conflicts of interest associated with this publication.

\section{Statement of informed consent, human/animal rights}

No conflicts, informed consent, human or animal rights applicable.

\section{Declaration of authors agreement to authorship}

The work described has not been published previously and it is not under consideration for publication elsewhere. The publication and submission of the manuscript for peer review is approved by all authors. 


\section{References}

542 [1] A.P. Carvalho, S.O. Silva, J.M. Baptista, F.X. Malcata, Light requirements in microalgal 543 photobioreactors: An overview of biophotonic aspects, Applied Microbiology and Biotechnology. $54489(2011) 1275-1288$.

545 [2] C. Wilhelm, T. Jakob, From photons to biomass and biofuels: Evaluation of different 546 strategies for the improvement of algal biotechnology based on comparative energy balances, 547 Applied Microbiology and Biotechnology. 92 (2011) 909-919.

548 [3] A. Richmond, Handbook of microalgal culture: biotechnology and applied phycology., 549 Blackwell Publishing Ltd, USA, 2004.

550 [4] C. Baroukh, R. Muñoz-Tamayo, J.-P. Steyer, O. Bernard, A state of the art of metabolic 551 networks of unicellular microalgae and cyanobacteria for biofuel production, Metabolic 552 Engineering. 30 (2015) 49-60.

553 [5] C. Posten, Design principles of photo-bioreactors for cultivation of microalgae, Engineering 554 in Life Sciences. 9 (2009) 165-177.

555 [6] M.A. Borowitzka, N.R. Moheimani, Algae for Biofuels and Energy, Springer, USA, 2013. 556 [7] D. Sutherland, V. Montemezzani, C. Howard-Williams, M. Turnbull, P. Broady, R. Craggs, 557 Modifying the high rate algal pond light environment and its effects on light absorption and 558 photosynthesis, Water Research. 70 (2015) 86-96.

559 [8] E. Molina-Grima, F.G. Acién Fernández, F. García Camacho, Y. Chisti, Photobioreactors: 560 light regime, mass transfer, and scaleup, Journal of Biotechnology. 70 (1999) 231-247.

561 [9] M. Henze, M.C.M. Van Loosdrecht, G.A. Ekama, D. Brdjanovic, Biological wastewater 
treatment : principles, modelling and design, IWA Publishing, UK, 2008.

[10] S.-K. Wang, A.R. Stiles, C. Guo, C.-Z. Liu, Microalgae cultivation in photobioreactors: An overview of light characteristics, Engineering in Life Sciences. 14 (2014) 550-559.

[11] H. Safafar, J. Van Wagenen, P. Møller, C. Jacobsen, Carotenoids, phenolic compounds and tocopherols contribute to the antioxidative properties of some microalgae species grown on industrial wastewater, Marine Drugs. 13 (2015) 7339-7356.

[12] M.J. Griffiths, C. Garcin, R.P. van Hille, S.T.L. Harrison, Interference by pigment in the estimation of microalgal biomass concentration by optical density, Journal of Microbiological Methods. 85 (2011) 119-123.

[13] J. Seyfabadi, Z. Ramezanpour, Z.A. Khoeyi, Protein, fatty acid, and pigment content of Chlorella vulgaris under different light regimes, Journal of Applied Phycology. 23 (2011) 721-726.

[14] V. Ördög, W.A. Stirk, P. Bálint, J. van Staden, C. Lovász, Changes in lipid, protein and pigment concentrations in nitrogen-stressed Chlorella minutissima cultures, Journal of Applied Phycology. 24 (2012) 907-914.

[15] X. Xie, A. Huang, W. Gu, Z. Zang, G. Pan, S. Gao, L. He, B. Zhang, J. Niu, A. Lin, G. Wang, Photorespiration participates in the assimilation of acetate in Chlorella sorokiniana under high light, New Phytologist. 209 (2016) 987-998.

[16] B. Araya, L. Gouveia, B. Nobre, A. Reis, R. Chamy, P. Poirrier, Evaluation of the simultaneous production of lutein and lipids using a vertical alveolar panel bioreactor for three Chlorella species, Algal Research. 6 (2014) 218-222.

[17] J.R. Benavente-Valdés, C. Aguilar, J.C. Contreras-Esquivel, A. Méndez-Zavala, J. 
583 Montañez, Strategies to enhance the production of photosynthetic pigments and lipids in 584 Chlorophycae species, Biotechnology Reports. 10 (2016) 117-125.

585 [18] Z. Rasouli, B. Valverde-Pérez, M. D’Este, D. De Francisci, I. Angelidaki, Nutrient recovery 586 from industrial wastewater as single cell protein by a co-culture of green microalgae and 587 methanotrophs, Biochemical Engineering Journal. 134 (2018) 129-135.

588 [19] Q. Béchet, A. Shilton, B. Guieysse, Modeling the effects of light and temperature on algae 589 growth: State of the art and critical assessment for productivity prediction during outdoor 590 cultivation, Biotechnology Advances. 31 (2013) 1648-1663.

591 [20] F. García-Camacho, A. Sánchez-Mirón, E. Molina-Grima, F. Camacho-Rubio, J.C. 592 Merchuck, A mechanistic model of photosynthesis in microalgae including photoacclimation 593 dynamics, Journal of Theoretical Biology. 304 (2012) 1-15.

594 [21] A.P. Koller, H. Löwe, V. Schmid, S. Mundt, D. Weuster-Botz, Model-supported 595 phototrophic growth studies with Scenedesmus obtusiusculus in a flat-plate photobioreactor, 596 Biotechnology and Bioengineering. 114 (2017) 308-320.

597 [22] O. Bernard, Hurdles and challenges for modelling and control of microalgae for $\mathrm{CO} 2$ 598 mitigation and biofuel production, Journal of Process Control. 21 (2011) 1378-1389.

599 [23] R.J. Geider, H.L. Maclntyre, T.M. Kana, A dynamic regulatory model of phytoplanktonic 600 acclimation to light, nutrients, and temperature, Limnol. Oceanogr. 43 (1998) 679-694.

601 [24] V.O. Adesanya, M.P. Davey, S.A. Scott, A.G. Smith, Kinetic modelling of growth and 602 storage molecule production in microalgae under mixotrophic and autotrophic conditions, 603 Bioresource Technology. 157 (2014) 293-304. 
604

605

606

607

608

609

610

611

612

613

614

615

616

617

618

619

620

621

622

623

624

[25] D.S. Wágner, B. Valverde-Pérez, M. Sæbø, M.B. de la Sotilla, J. Van Wagenen, B.F. Smets, B.G. Plósz, Towards a consensus-based biokinetic model for green microalgae - the ASM-A, Water Research. 103 (2016) 485-499.

[26] W. Blanken, P.R. Postma, L. de Winter, R.H. Wijffels, M. Janssen, Predicting microalgae growth, Algal Research. 14 (2016) 28-38.

[27] X. Wu, J.C. Merchuk, Simulation of algae growth in a bench scale internal loop airlift reactor, Chemical Engineering Science. 59 (2004) 2899-2912.

[28] J. Van Wagenen, M.L. Pape, I. Angelidaki, Characterization of nutrient removal and microalgal biomass production on an industrial waste-stream by application of the deceleration-stat technique, Water Research. 75 (2015) 301-311.

[29] M. Huesemann, B. Crowe, P. Waller, A. Chavis, S. Hobbs, S. Edmundson, M. Wigmosta, A validated model to predict microalgae growth in outdoor pond cultures subjected to fluctuating light intensities and water temperatures, Algal Research. 13 (2016) 195-206.

[30] R.R.L. Guillard, C.J. Lorenzen, Yellow-green algae with chlorophyllide C, Journal of Phycology. 8 (1972) 10-14.

[31] B. Valverde-Pérez, Wastewater resource recovery via the Enhanced Biological Phosphorus Removal and Recovery (EBP2R) process coupled with green microalgae cultivation, Technical University of Denmark, Kgs. Lyngby, Denmark, 2015.

[32] APHA, American Water Works Association, Water Environment Federation, Standard methods for the examination of water and wastewater, Washington DC, 1999.

[33] J. Van Wagenen, D. De Francisci, I. Angelidaki, Comparison of mixotrophic to cyclic 
625 626 627 628 629 630 631 632 633 634 635 636 637 638 639 640 641 642 643 644 645 646

autotrophic/heterotrophic growth strategies to optimize productivity of Chlorella sorokiniana, Journal of Applied Phycology. 27 (2015) 1775-1782.

[34] C. Paliwal, T. Ghosh, B. George, I. Pancha, R. Maurya, K. Chokshi, A. Ghosh, S. Mishra, Microalgal carotenoids: Potential nutraceutical compounds with chemotaxonomic importance, Algal Research. 15 (2016) 24-31.

[35] J.F. Cornet, C.G. Dussap, P. Cluzel, G. Dubertret, A structured model for simulation of cultures of the cyanobacterium Spirulina platensis in photobioreactors: II. Identification of kinetic parameters under light and mineral limitations., Biotechnology and Bioengineering. 40 (1992) 826834.

[36] V.S. Ferreira, R.F. Pinto, C. Sant'Anna, Low light intensity and nitrogen starvation modulate the chlorophyll content of Scenedesmus dimorphus, Journal of Applied Microbiology. 120 (2015) 661-670.

[37] H. Akaike, Information theory and an extension of the maximum likelihood principle, in: International Symposium on Information Theory, 1973: pp. 267-281.

[38] G. Sin, A.S. Meyer, K. V. Gernaey, Assessing reliability of cellulose hydrolysis models to support biofuel process design-Identifiability and uncertainty analysis, Computers and Chemical Engineering. 34 (2010) 1385-1392.

[39] C.B.S. Dotto, G. Mannina, M. Kleidorfer, L. Vezzaro, M. Henrichs, D.T. McCarthy, G. Freni, W. Rauch, A. Deletic, Comparison of different uncertainty techniques in urban stormwater quantity and quality modelling, Water Research. 46 (2012) 2545-2558.

[40] P. Ramin, B. Valverde-Pérez, F. Polesel, L. Locatelli, B.G. Plósz, A systematic model identifcation method for chemical transformation pathways - the case of heroin biomarkers in 
647

648

649

650

651

652

653

654

655

656

657

658

659

660

661

662

663

664

665

666

667

wastewater, Scientific Reports. 7 (2017) 9390.

[41] R. Pandey, A. Sahu, V.K. K, P. M, Studies on light intensity distribution inside an open pond photo-bioreactor, Bioprocess and Biosystems Engineering. 38 (2015) 1547-1557.

[42] I. Vaquero, B. Mogedas, M.C. Ruiz-Domínguez, J.M. Vega, C. Vílchez, Light-mediated lutein enrichment of an acid environment microalga, Algal Research. 6 (2014) 70-77.

[43] L.L. Fang, B. Valverde-Pérez, A. Damgaard, B.G. Plósz, M. Rygaard, Life cycle assessment as development and decision support tool for wastewater resource recovery technology, Water Research. 88 (2016) 538-549.

[44] S. Van Den Hende, E. Carré, E. Cocaud, V. Beelen, N. Boon, H. Vervaeren, Treatment of industrial wastewaters by microalgal bacterial flocs in sequencing batch reactors, Bioresource Technology. 161 (2014) 245-254.

[45] N. Aburai, D. Sumida, K. Abe, Effect of light level and salinity on the composition and accumulation of free and ester-type carotenoids in the aerial microalga Scenedesmus sp.

(Chlorophyceae), Algal Research. 8 (2015) 30-36.

[46] F. Guihéneuf, D.B. Stengel, Towards the biorefinery concept: Interaction of light, temperature and nitrogen for optimizing the co-production of high-value compounds in Porphyridium purpureum, Algal Research. 10 (2015) 152-163.

[47] M. D’Este, D. De Francisci, I. Angelidaki, Novel protocol for lutein extraction from microalga Chlorella vulgaris, Biochemical Engineering Journal. 127 (2017) 175-179.

[48] R. Geider, J. La Roche, Redfield revisited: variability of C:N:P in marine microalgae and its biochemical basis, European Journal of Phycology. 37 (2002) 1-17. 

669 physiology and metabolism of Chlorella vulgaris var L3, Algal Research. 10 (2015) 134-144.

670 [50] A. Liu, A. Goonetilleke, P. Egodawatta, Role of rainfall and catchment characteristics on 671 urban stormwater quality, Springer Singapore, 2015.

672 [51] H. Hauduc, M.B. Neumann, D. Muschalla, V. Gamerith, S. Gillot, P.A. Vanrolleghem, 673 Efficiency criteria for environmental model quality assessment: A review and its application to 674 wastewater treatment, Environmental Modelling \& Software. 68 (2015) 196-204.

675 [52] J. Van Wagenen, S.L. Holdt, D. De Francisci, B. Valverde-Pérez, B.G. Plósz, I. Angelidaki, 676 Microplate-based method for high-throughput screening of microalgae growth potential, 677 Bioresource Technology. 169 (2014) 566-572.

678 679 
681
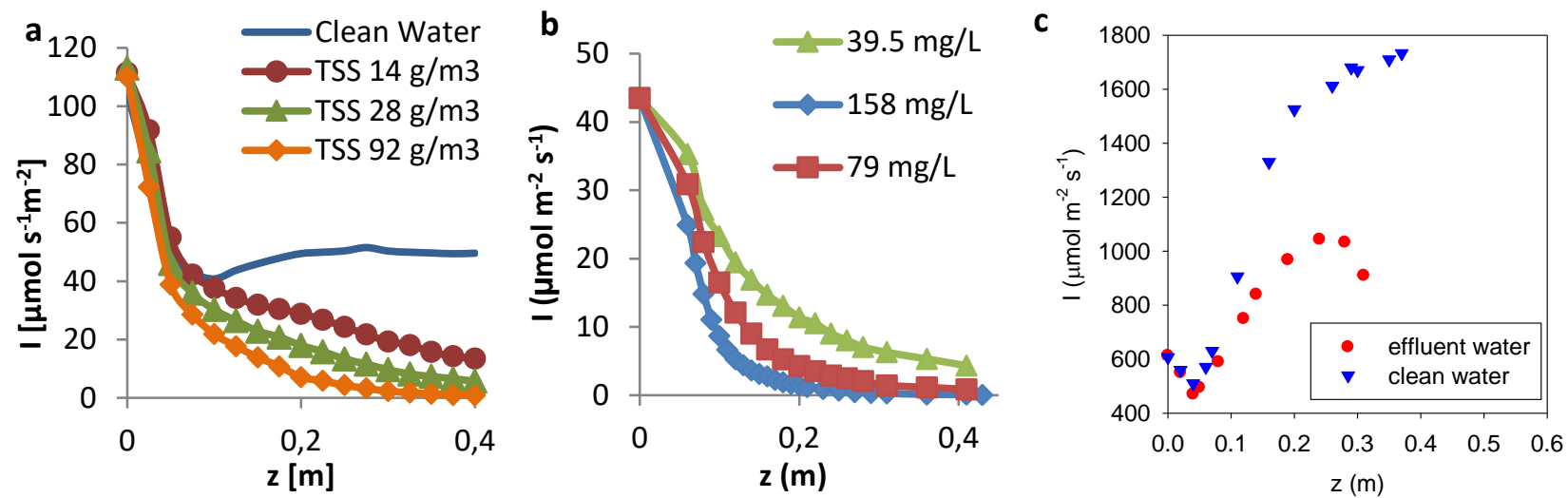

683 Figure 1: Light attenuation and scattering in photobioreactors, PBR (reactor diameter: $0.14 \mathrm{~m}$ ) - effects of 684 scattering on light distribution in PBRs. Due to the scattering on the reactor walls, the light intensity 685 increases towards the bottom of the reactor - the bottom and the sides of the reactor were both covered with 686 black cloth, thus light only entered from the top of the reactor. (a) Light attenuation inside the PBRs at 687 different biomass concentrations, with nutrient-limited cultivation, and in clean water; (b) Light attenuation 688 inside the PBR at different biomass concentrations with nutrients in excess cultivation; (c) Light attenuation 689 inside the PBR with clean water and effluent used water. 

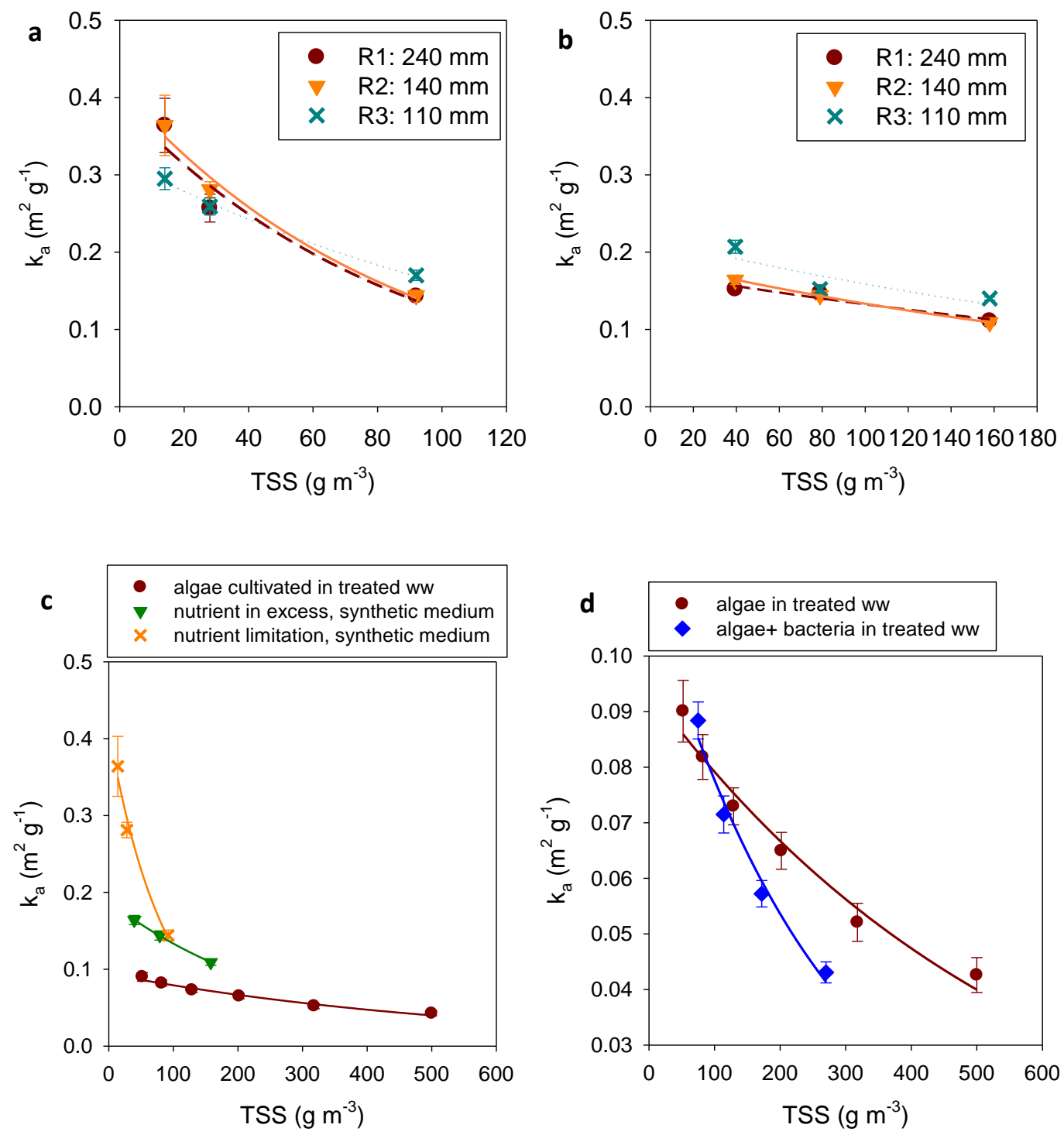

693 Figure 2: Estimation of the attenuation coefficient $\left(\mathrm{k}_{\mathrm{a}}\right)$ values in PBRs with three different diameters and at 694 three different biomass concentrations. The estimation of $k_{a}$ values was done both at (a) nutrient limited 695 conditions and at (b) nutrients-in-excess conditions (see Fig. S6, SI where the different pigmentations are 696 shown). The lines show exponential regression functions fitted on the measured data sets (values of 697 regression coefficients shown in Table S4). The dashed red line shows the fitting for R1, the solid orange 698 line shows the fitting for R2 and the dotted blue line shows the fitting for R3. (c) Values of $k_{a}$ obtained at 699 different biomass concentrations with algae cultivated in synthetic medium and EBPR process effluent water 700 (denoted as ww in the legend). The observations were made in Reactor 2 (140 mm diameter). (d) The effect 
701 of increased bacterial biomass concentration on the light attenuation in the PBR. TSS in this figure represents 702 the total TSS of algal + bacterial biomass where the amount of bacteria was increased whilst algal biomass 703 was kept constant (at $75 \mathrm{mg} / \mathrm{L}$ ). The observations were made in Reactor 2 (140 mm diameter). The error bars 704 present the standard error of the estimate parameter value obtained through regression in SigmaPlot ${ }^{\circ}$ 

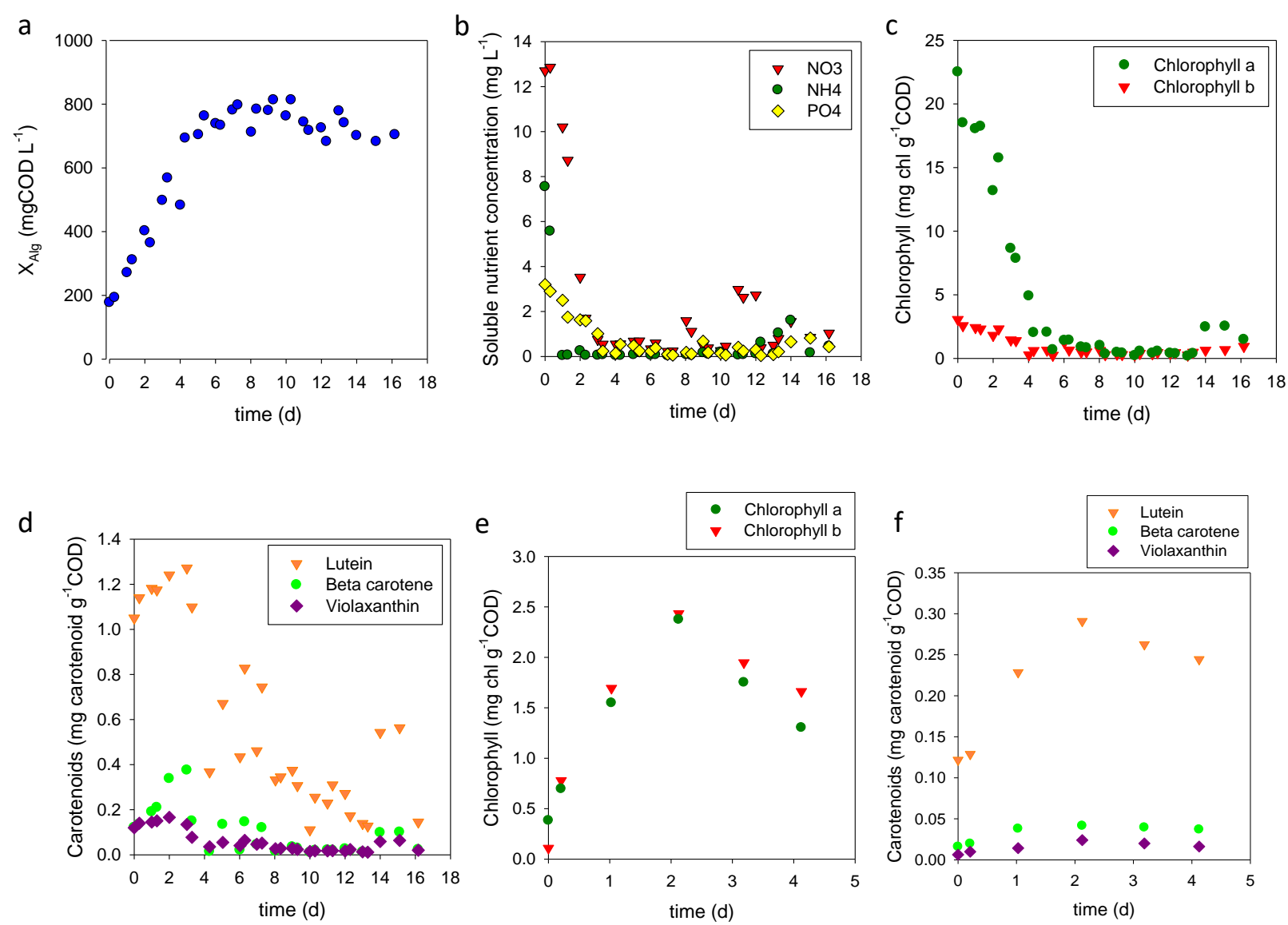

Figure 3: Batch algal cultivation. (a) Microalgal biomass growth during the batch cultivation where 709 nutrients were added to a dilute culture ( $185 \mathrm{mg}$ COD/L initial algal biomass) at day 0 and were depleted by 710 day 3 (Batch 1). (b) Nitrogen and phosphorus concentration during cultivation in Batch 1. (c) Chlorophyll $a$ 711 and $b$ and (d) carotenoids concentrations obtained in Batch 1. (e) Chlorophyll $a$ and $b$ and (f) carotenoids 712 concentration obtained in batch cultivation in Batch 2 where nutrients were added to a dense (400 mg $713 \mathrm{COD} / \mathrm{L}$ initial biomass concentration) and highly nutrient limited culture at day 0 and were depleted by day 2 
a

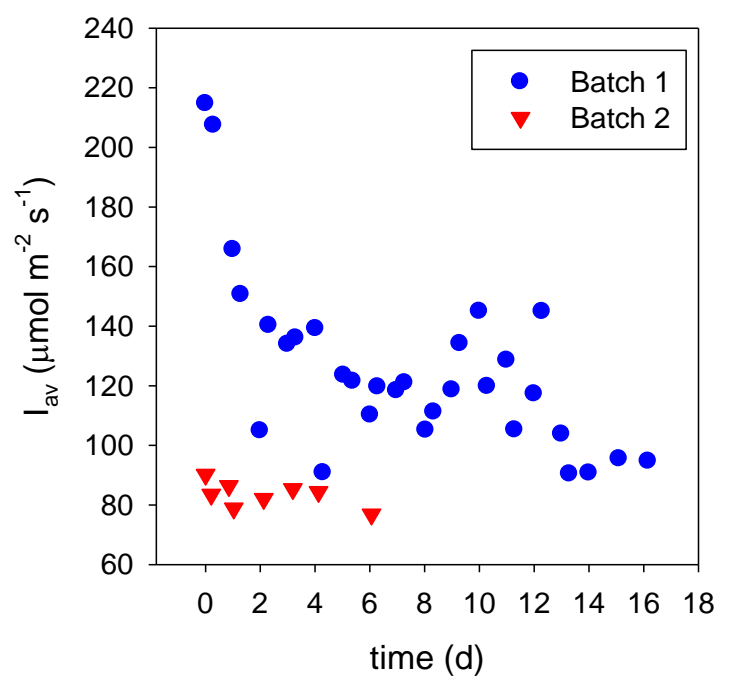

b

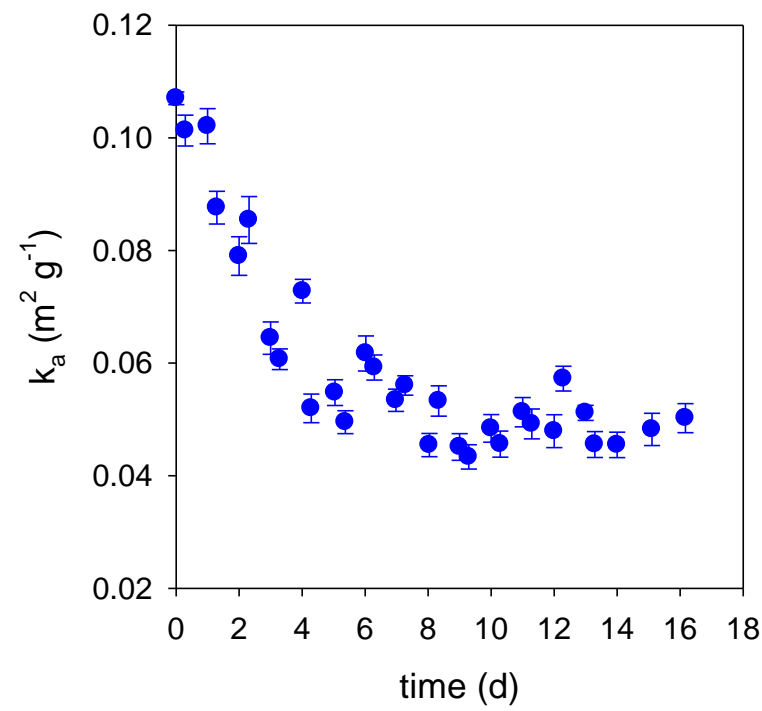

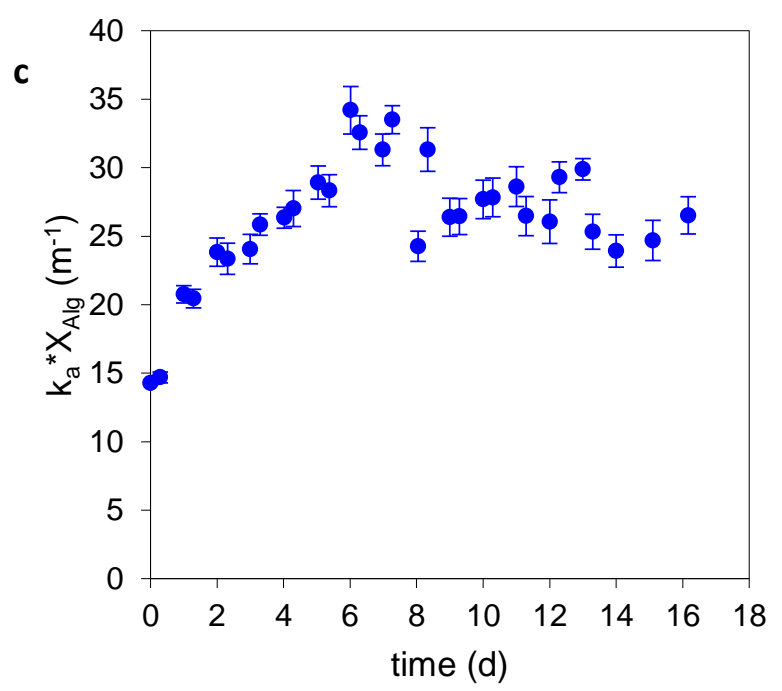

$717 \quad$ Figure 4: Light intensity and attenuation in Batch 1 and 2. (a) Average light intensity in the reactor during Batch 1 and Batch 2 cultivation. The average light intensity was calculated by integrating the Lambert-Beer equation at each time step. (b) Variation of the light attenuation coefficient $\left(\mathrm{k}_{\mathrm{a}}\right)$ over time during the batch cultivation (in Batch 1). Values of $k_{a}$ were estimated by measuring the light intensity at different depths of 721 the reactor and fitting the Lambert-Beer equation. (c) Variation of the effective attenuation coefficient, 722 calculated by the product of $\mathrm{k}_{\mathrm{a}}$ and the biomass concentration $\left(\mathrm{X}_{\mathrm{Alg}}\right)$. The error bars present the standard error 723 of the estimate parameter value obtained through regression in SigmaPlot ${ }^{\circledR}$. 


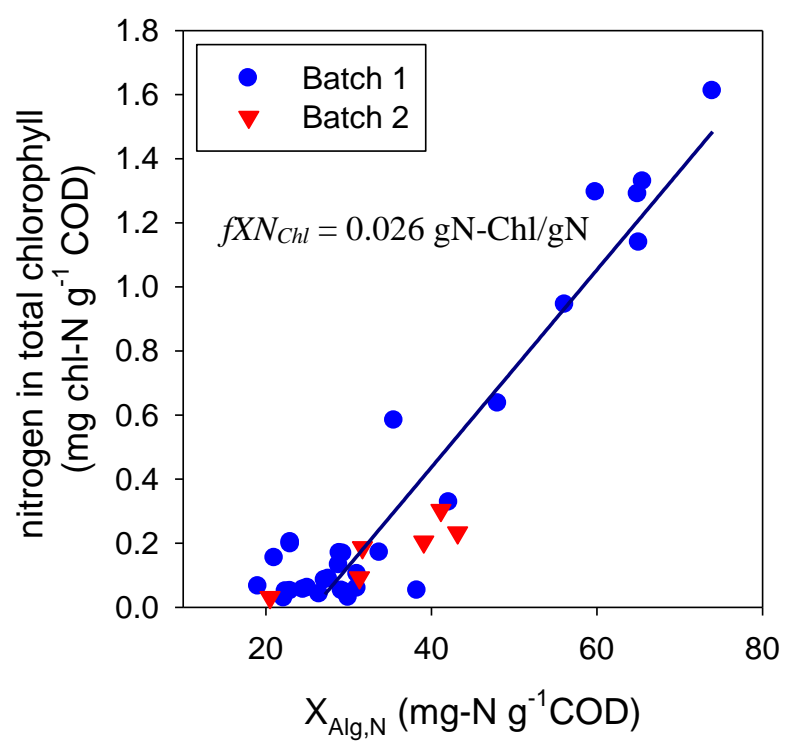

727 Figure 5: The nitrogen content of total chlorophyll expressed as Chl-N plotted against the internal nitrogen 728 quota. The fraction of chlorophyll-nitrogen $\left(\mathrm{fXN}_{\mathrm{Chl}}\right)$ to the total cellular nitrogen quota was estimated from 729 the slope. 

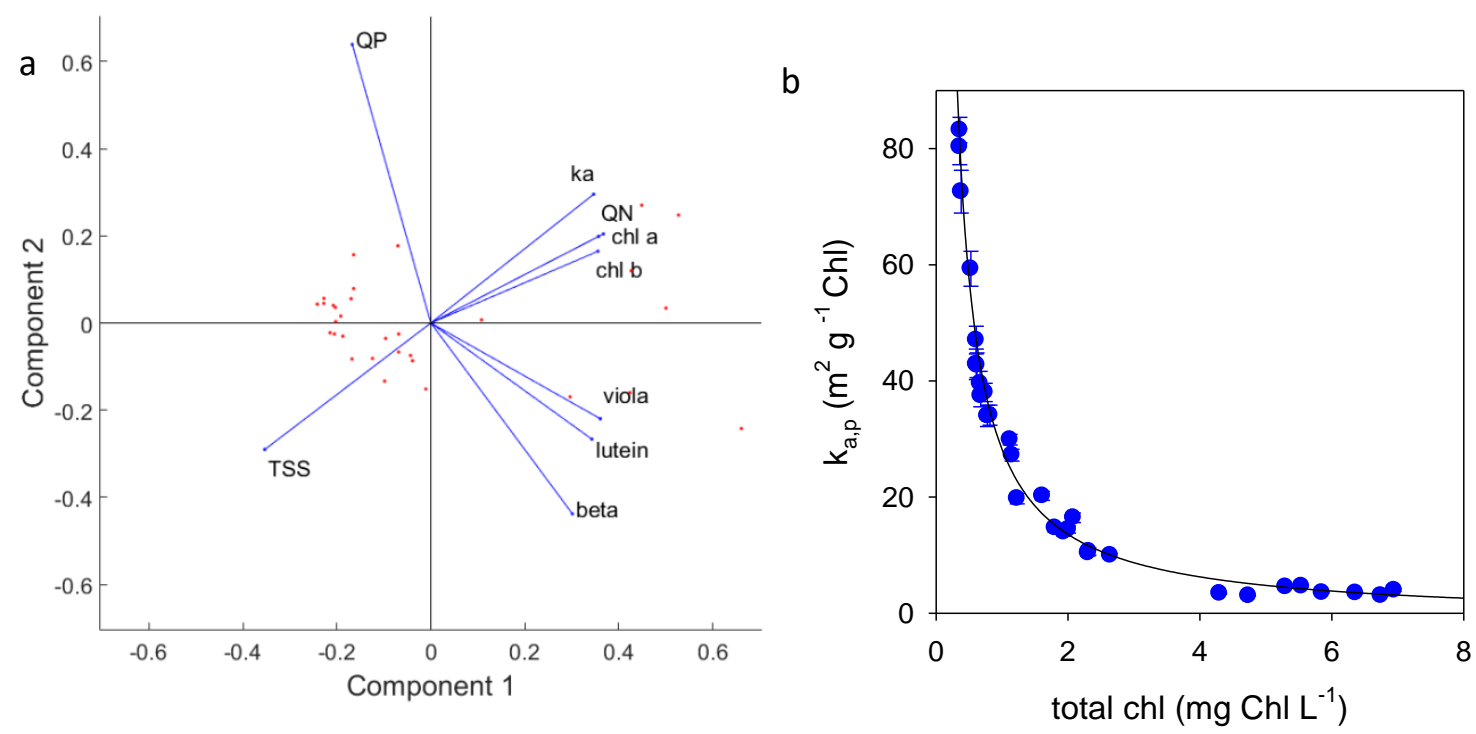

732 Figure 6: Light attenuation and prediction of $k_{a}$ in PBR. (a) PCA analysis showing the factors that can 733 affect the light attenuation. (b) Estimation of the attenuation coefficient specific for the chlorophyll 734 content in Batch 1. The error bars present the standard error of the estimate parameter value obtained through 735 regression in SigmaPlot ${ }^{\circledR}$. 


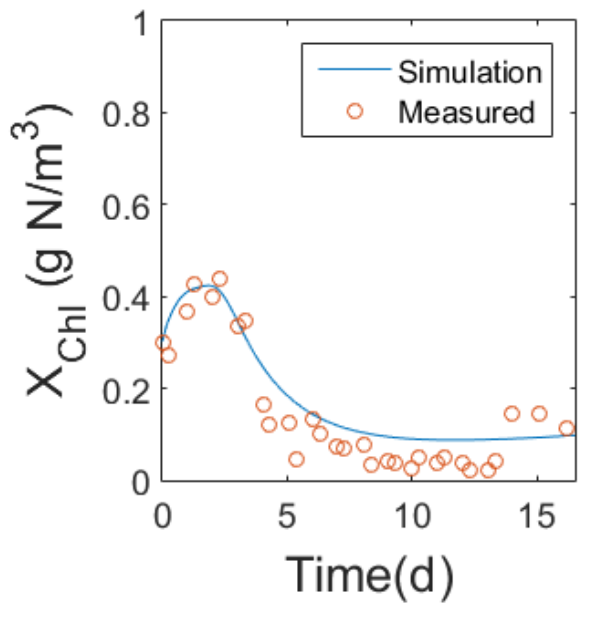

738 Figure 7: Simulation of batch experimental data using the extended ASM-A model. Prediction of

739 the chlorophyll content of the microalgae in Batch 1. 

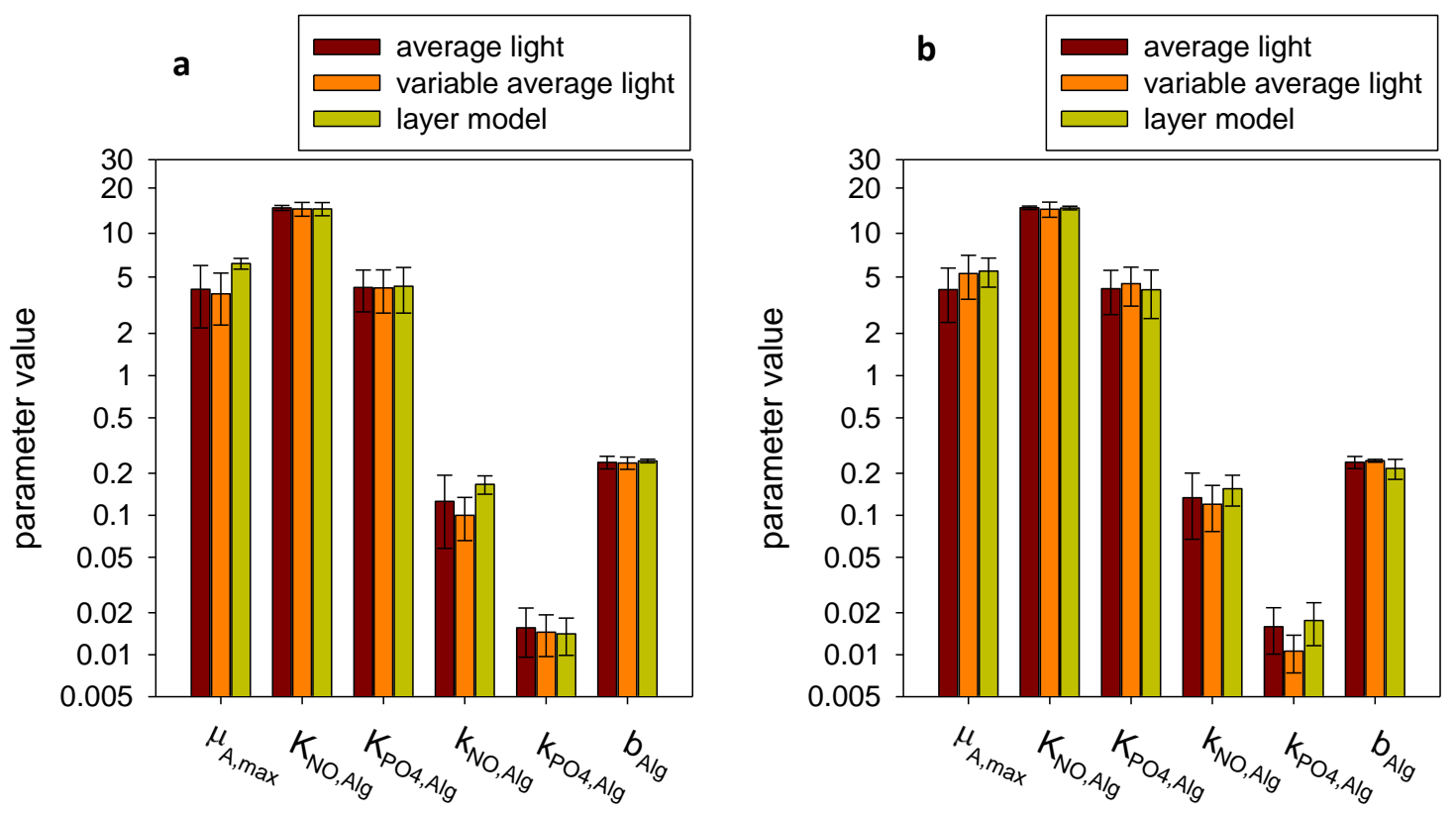

742 Figure 8: Comparison of the estimated parameter (mean value and 95\% confidence interval) values 743 using different model complexity levels (CL1 - CL3). On the y-axis Weibull type scaling is used to 744 allow comparison of parameter values at different scales. (a) The TSS is used to calculate the 745 attenuation coefficient $\left(\mathrm{k}_{\mathrm{a}}\right)$. (b) The simulation model extended to predict the algal chlorophyll 746 content is used to estimate the pigment specific attenuation coefficient $\left(\mathrm{k}_{\mathrm{a}, \mathrm{p}}\right)$. 



750 Figure 9: Simulation of batch experimental data (Batch 1) using the extended ASM-A implemented

751 as CL- 3 (one-dimensional layer model) with the mean values of the parameters estimated. The 752 uncertainty bands are shown in blue. The chlorophyll content is used to calculate the pigment 753 specific attenuation coefficient $\left(\mathrm{k}_{\mathrm{a}, \mathrm{p}}\right)$ that is used in the simulations to predict the light intensity. 
Table 1: The Gujer matrix of ASM-A model including the state-variables, the stoichiometric coefficients and the process rate equations identified in [25]. The grey highlighted columns and rows include the model extension presented in this paper to estimate the chlorophyll content.

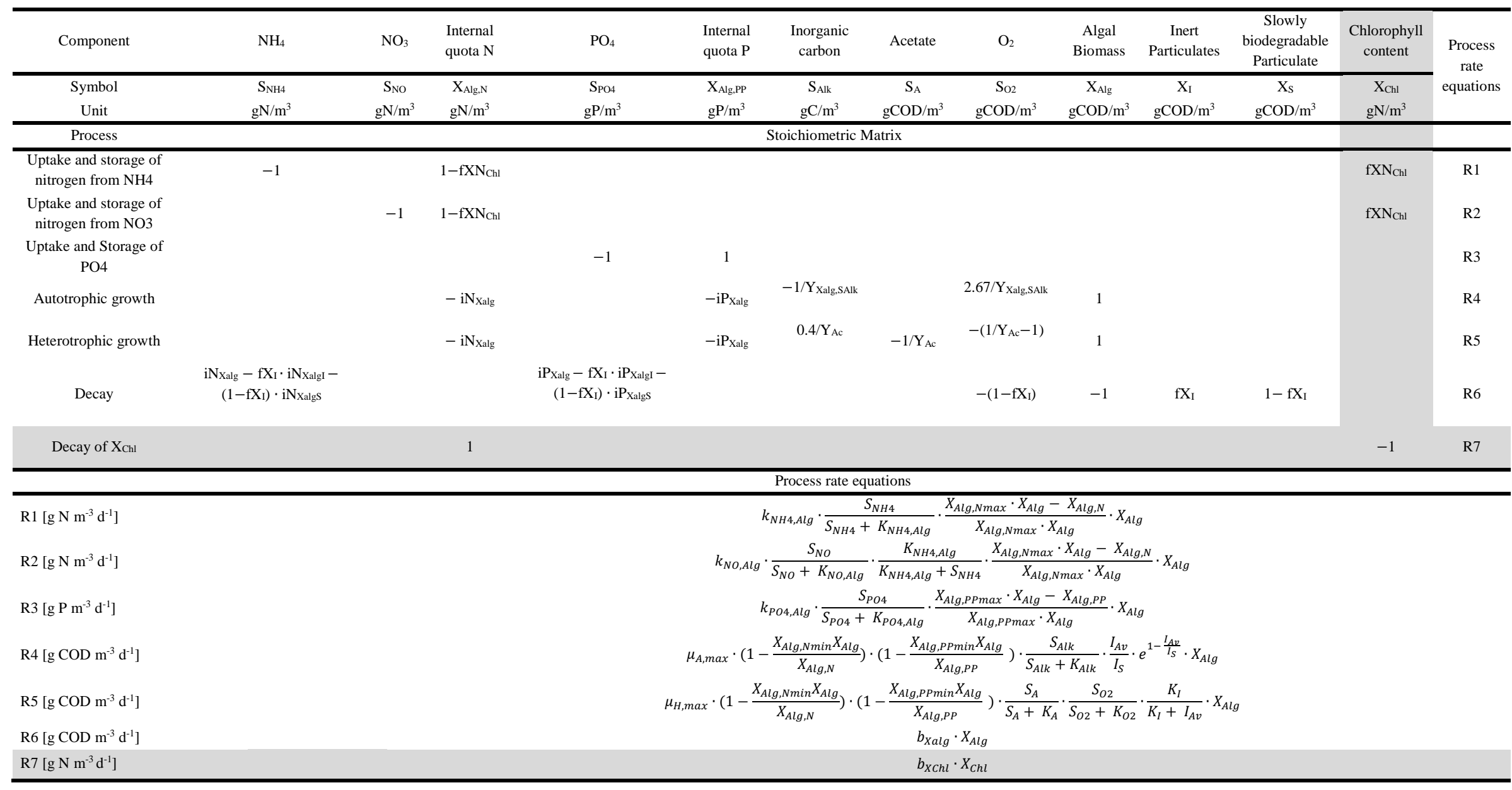




\section{Supporting Information}

"Light attenuation in photobioreactors and algal pigmentation under different growth conditions - model identification and complexity assessment"

Dorottya S. Wágner ${ }^{\mathrm{a}, \mathrm{b}, *}$, Borja Valverde-Pérez ${ }^{\mathrm{a}}$, Benedek Gy. Plósz, ${ }^{\mathrm{a}, \mathrm{c}, *}$

a Department of Environmental Engineering, Technical University of Denmark, Miljфvej, Building 115, 2800 Kgs. Lyngby, Denmark

${ }^{\mathrm{c}}$ Department of Chemical Engineering, University of Bath, Claverton Down, Bath BA2 7AY, UK

*Corresponding authors: dsw@bio.aau.dk; bgp24@bath.ac.uk

The Supporting Information consists of 29 pages. It contains 14 tables (pages 6-16) and 19 figures (pages 17-28). 


\section{$\underline{\text { SI-1 Measurement of light distribution in three reactors with different diameters }}$}

First, a blank test was carried out, where light intensity was measured over depth in reactors filled with clean tap water. The effect of aeration with different bubble size was assessed. Three different diffusers were tested during the experiments. Bubble size was measured manually based on pictures taken during the experiments, by relating the bubble size to the size of the diffuser.

Microalgae were cultivated in the effluent water of a laboratory-scale EBPR system (as described in section 2.1), to assess the effect of effluent water on the light attenuation in the reactor. A blank test was carried out to assess the light attenuation in the reactor in effluent water, without the addition of algae. Moreover, bacterial biomass was taken from the EBPR system and was spiked in the reactor containing microalgae cultivated in the effluent water to assess its impact on the light attenuation. Biomass concentrations used in each experiment are reported in Table S1. 


\section{$\underline{\text { SI-2 Pigment extraction protocol }}$}

$1 \mathrm{ml}$ of microalgae sample was collected in $1.5 \mathrm{ml}$ Eppendorf tube and centrifuged for $10 \mathrm{~min}$ at $10000 \mathrm{rpm}$. The pellet was kept at $-20^{\circ} \mathrm{C}$ until the extraction. The pigment extraction was done in darkness using green light to minimize the degradation of extracted pigments and when possible keeping them in ice. $1 \mathrm{ml} 99.9 \%$ HPLC grade methanol (Sigma-Aldrich, Germany) was added to the pellets and mixed with vortex. Ultrasonic bath (Retsch U1, Germany) was used to break the microalgal cells. During the sonication the samples were cooled with ice. Following the 60 min sonication the samples were kept on ice for $30 \mathrm{~min}$ to enhance extraction of pigments. The samples were then centrifuged for $5 \mathrm{~min}$ at $10000 \mathrm{rpm}$. The supernatant was filtered through $0.2 \mu \mathrm{m}$ syringe filters (Agilent Technologies, USA) and $200 \mu 1$ filtered sample was mixed with $600 \mu 128 \mathrm{mM}$ Tetrabutylammoniumacetate buffer solution in amber glass vials. The samples were placed in the UHPLC and were cooled at $8{ }^{\circ} \mathrm{C}$ until analysis. 


\section{$\underline{\text { SI-3 Model evaluation criteria }}$}

The RMSNE was calculated as:

$$
R M S N E=\sqrt{\frac{1}{n} \sum_{i=1}^{n}\left(\frac{y_{m}-y}{y_{m}}\right)^{2}}
$$

where $\mathrm{n}$ is the number of measurement points, $\mathrm{y}_{\mathrm{m}}$ is the measured value and $\mathrm{y}$ is the predicted value. The AIC criterion is estimated by Akaike (1973):

$A I C=N * \ln \left(\frac{S S}{N}\right)+2 * K \quad$ Eq. $\mathrm{S} 2$

where $\mathrm{N}$ is the number of data points, $\mathrm{SS}$ is the sum of squares of the difference between the measured data and model prediction, $\mathrm{K}$ is the number of parameters estimated plus one. This criteria indicates the goodness of fit of the model predictions, where a lower AIC suggests better fit. Mean and $95 \%$ confidence interval of the estimated parameter subsets were compared in the second criterion, and the parameter correlation in the third criterion, thereby assessing the impact of model structure on parameter identifiability based on the LHSS output. Finally, in the fourth criterion, the model prediction uncertainties were compared. Monte Carlo simulations were performed to obtain a confidence interval of model predictions (Sin et al., 2009). The uncertainty classes were assigned to each parameter based on Wágner et al. (2016). The probability range of the estimated parameters was calculated by the mean and the $95 \%$ confidence interval. $1000 \mathrm{MC}$ simulations were run as specified by Wágner et al. (2016).

ARIL is calculated based on (Dotto et al., 2012):

$A R I L=\frac{1}{N} \sum_{i=1}^{N} \frac{\text { Limit }_{\text {upper }, i}-\text { Limit }_{\text {lower }, i}}{X_{o b s, i}}$ Eq. S3 


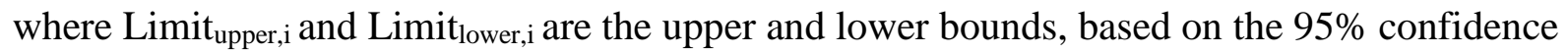
interval obtained in the Monte Carlo simulations, $\mathrm{X}_{\mathrm{obs}, \mathrm{i}}$ is the measured value, $\mathrm{N}$ is the number of measurement points. ARIL is used in combination with the coverage, which is the percentage of the observations that are within the prediction bands. Lower ARIL and a higher coverage suggest better model performance. Ramin et al. (2016) expressed the combination of the two evaluation criteria:

$A R I L C=\frac{A R I L}{\text { coverage }} \quad$ Eq. S4

where a smaller ARILC indicates better model prediction. 
$\underline{\text { Tables }}$

Table S1: Initial conditions of the experiments used to assess the light distribution in three different reactors (R1, R2 and R3) at different biomass concentrations.

\begin{tabular}{|c|c|c|c|c|c|c|c|}
\hline & & \multicolumn{2}{|c|}{$\mathrm{R} 1(240 \mathrm{~mm})$} & \multicolumn{2}{|c|}{$\mathrm{R} 2(140 \mathrm{~mm})$} & \multicolumn{2}{|c|}{$\mathrm{R} 3(110 \mathrm{~mm})$} \\
\hline & $\begin{array}{c}\mathrm{X}_{\mathrm{Alg}} \\
(\mathrm{mg} / \mathrm{L})\end{array}$ & $\begin{array}{c}\mathrm{I}_{0} \\
\left(\mu \mathrm{mol} / \mathrm{m}^{2} / \mathrm{s}\right)\end{array}$ & $\begin{array}{l}X_{\text {bacteria }} \\
(\mathrm{mg} / \mathrm{L})\end{array}$ & $\begin{array}{c}\mathrm{I}_{0} \\
\left(\mu \mathrm{mol} / \mathrm{m}^{2} / \mathrm{s}\right)\end{array}$ & $\begin{array}{l}\mathrm{X}_{\text {bacteria }} \\
(\mathrm{mg} / \mathrm{L})\end{array}$ & $\begin{array}{c}\mathrm{I}_{0} \\
\left(\mu \mathrm{mol} / \mathrm{m}^{2} / \mathrm{s}\right)\end{array}$ & $\begin{array}{l}X_{\text {bacteria }} \\
(\mathrm{mg} / \mathrm{L})\end{array}$ \\
\hline \multirow{3}{*}{$\begin{array}{l}\text { nutrient limited } \\
\text { cultivation }\end{array}$} & 14 & 104 & & 112 & & 229 & \\
\hline & 28 & 112 & & 113 & & 234 & \\
\hline & 92 & 112 & & 110 & & 266 & \\
\hline \multirow{3}{*}{$\begin{array}{l}\text { nutrients in excess } \\
\text { cultivation }\end{array}$} & 39.5 & 42 & & 44 & & 353 & \\
\hline & 79 & 42 & & 44 & & 353 & \\
\hline & 158 & 38 & & 44 & & 353 & \\
\hline \multirow{6}{*}{$\begin{array}{l}\text { cultivation in used } \\
\text { water resources }\end{array}$} & 52 & & & 1032 & & & \\
\hline & 82 & & & 1021 & & & \\
\hline & 129 & & & 1054 & & & \\
\hline & 202 & & & 1087 & & & \\
\hline & 318 & & & 975 & & & \\
\hline & 500 & & & 1170 & & & \\
\hline \multirow{4}{*}{ addition of bacteria } & 75 & & & 1099 & 0 & & \\
\hline & 75 & & & 1059 & 39 & & \\
\hline & 75 & & & 1068 & 97 & & \\
\hline & 75 & & & 1281 & 195 & & \\
\hline
\end{tabular}


Table S2: The estimated attenuation coefficients (based on the Lambert-Beer equation) for the nutrient limited and nutrients in excess cultivation in three different reactor diameters and six biomass concentrations. The blue shading refers to scenarios where the estimated attenuation coefficient is equal (or not significantly different from) to the $E_{a}$ estimated by the Schuster's law.

\begin{tabular}{|c|r|c|c|c|}
\cline { 2 - 5 } \multicolumn{1}{c|}{} & \multirow{2}{*}{$\begin{array}{c}\text { TSS } \\
(\mathrm{mg} / \mathrm{l})\end{array}$} & \multicolumn{4}{|c|}{ Diameter $(\mathrm{mm})$} \\
\cline { 3 - 5 } & & 240 & 140 & 110 \\
\cline { 3 - 5 } & $\mathrm{k}_{\mathrm{a}}$ & $\begin{array}{c}\mathrm{k}_{\mathrm{a}} \\
\left(\mathrm{m}^{2} / \mathrm{g}\right)\end{array}$ & $\begin{array}{c}\mathrm{k}_{\mathrm{a}} \\
\left(\mathrm{m}^{2} / \mathrm{g}\right)\end{array}$ \\
\hline \multirow{3}{*}{ nutrient limited } & 14 & 0.364 & 0.364 & 0.295 \\
\cline { 2 - 5 } & 28 & 0.257 & 0.281 & 0.259 \\
\cline { 2 - 5 } & 92 & 0.14 & 0.144 & 0.17 \\
\hline \multirow{3}{*}{ nutrients in excess } & 39.5 & 0.15 & 0.16 & 0.207 \\
\cline { 2 - 5 } & 79 & 0.15 & 0.14 & 0.152 \\
\cline { 2 - 5 } & 158 & 0.11 & 0.11 & 0.14 \\
\hline
\end{tabular}

Table S3: Light parameters $a$ and $b$ estimated (average \pm standard deviation) for the nutrient limited, nutrients in-excess cultivation in synthetic medium and cultivation in treated water. Constitutive relation for light attenuation: $k_{a}=a * e^{-b * X_{A l g}}$.

\begin{tabular}{|l|l|l|l|}
\cline { 2 - 4 } \multicolumn{1}{c|}{} & Nutrient limited & Nutrient in-excess & Treated water \\
\hline$a\left(\mathrm{~m}^{2} / \mathrm{g}\right)$ & $0.374 \pm 0.029$ & $0.194 \pm 0.0079$ & $0.094 \pm 0.003$ \\
\hline$b\left(\mathrm{~g} / \mathrm{m}^{3}\right)$ & $0.01 \pm 0.0017$ & $0.0031 \pm 0.0004$ & $0.0017 \pm 0.0001$ \\
\hline
\end{tabular}


Table S4: In-reactor light path length multiplier (PLM) calculated for the nutrient limited and nutrients in excess scenarios. Curve fitting was done by using the estimated $k_{a}$ in the two scenarios and the Lambert-Beer equation. PLM was used to improve fit (based on $\mathrm{R}^{2}$ ). The blue shading refers to scenarios where PLM is not needed.

\begin{tabular}{|c|c|c|c|c|c|c|c|c|c|c|}
\hline & \multirow{3}{*}{ TSS (mg/l) } & \multicolumn{3}{|c|}{ diameter $(\mathrm{mm})$} & \multicolumn{3}{|c|}{ diameter $(\mathrm{mm})$} & \multicolumn{3}{|c|}{ diameter $(\mathrm{mm})$} \\
\hline & & 240 & 140 & 110 & 240 & 140 & 110 & 240 & 140 & 110 \\
\hline & & \multicolumn{3}{|c|}{ PLM (-) } & \multicolumn{3}{|c|}{$\mathrm{R}^{2}$ of fit without PLM } & \multicolumn{3}{|c|}{$\mathrm{R}^{2}$ of fit with PLM } \\
\hline \multirow{3}{*}{ nutrient limited } & 14 & 1.4 & 1.6 & 1.4 & 0.51 & 0.04 & 0.5 & 0.82 & 0.7 & 0.74 \\
\hline & 28 & 1.1 & 1.3 & 1.3 & 0.86 & 0.69 & 0.79 & 0.88 & 0.83 & 0.87 \\
\hline & 92 & 1 & 1.1 & 1.3 & 0.97 & 0.92 & 0.87 & 0.97 & 0.96 & 0.95 \\
\hline \multirow{3}{*}{$\begin{array}{l}\text { nutrients in } \\
\text { excess }\end{array}$} & 39.5 & 1 & 1 & 1.6 & 0.93 & 0.97 & 0.33 & 0.93 & 0.97 & 0.86 \\
\hline & 79 & 1 & 1 & 1.1 & 0.93 & 0.94 & 0.9 & 0.93 & 0.94 & 0.96 \\
\hline & 158 & 1 & 1 & 1.2 & 0.93 & 0.95 & 0.91 & 0.93 & 0.95 & 0.99 \\
\hline
\end{tabular}


Table S5: The Lambert-Beer equation is fitted on the light curves and their fit is compared to the fitting with Schuster's law. The comparison is based on $\mathrm{R}^{2}$ of the fit. The blue shading refers to scenarios where scattering is not relevant thus Lambert-Beer equation and Shuster's law give the same fitting.

\begin{tabular}{|c|c|c|c|c|c|c|c|}
\hline & \multirow{3}{*}{$\begin{array}{c}\text { TSS } \\
(\mathrm{mg} / \mathrm{l})\end{array}$} & \multicolumn{6}{|c|}{ Diameter $(\mathrm{mm})$} \\
\hline & & \multicolumn{2}{|c|}{240} & \multicolumn{2}{|c|}{140} & \multicolumn{2}{|c|}{110} \\
\hline & & $\begin{array}{c}E_{a} \\
\left(\mathrm{~m}^{2} / g\right)\end{array}$ & $\begin{array}{c}\mathrm{E}_{\mathrm{s}} \\
\left(\mathrm{m}^{2} / \mathrm{g}\right)\end{array}$ & $\begin{array}{c}\mathrm{E}_{\mathrm{a}} \\
\left(\mathrm{m}^{2} / \mathrm{g}\right)\end{array}$ & $\begin{array}{c}\mathrm{E}_{\mathrm{s}} \\
\left(\mathrm{m}^{2} / \mathrm{g}\right)\end{array}$ & $\begin{array}{c}E_{a} \\
\left(\mathrm{~m}^{2} / \mathrm{g}\right)\end{array}$ & $\begin{array}{c}\mathrm{E}_{\mathrm{s}} \\
\left(\mathrm{m}^{2} / \mathrm{g}\right)\end{array}$ \\
\hline \multirow{3}{*}{ nutrient limited } & 14 & 0.003 & 1.86 & 0.01 & 2.4 & 0.017 & 2.35 \\
\hline & 28 & 0.019 & 1.26 & 0.016 & 1.63 & 0.023 & 1.64 \\
\hline & 92 & 0.14 & 0 & 0.025 & 0.53 & 0.026 & 0.64 \\
\hline \multirow{3}{*}{$\begin{array}{l}\text { nutrients in } \\
\text { excess }\end{array}$} & 39.5 & 0.15 & 0 & 0.16 & 0 & 0.013 & 1.2 \\
\hline & 79 & 0.14 & 0 & 0.14 & 0 & 0.021 & 0.64 \\
\hline & 158 & 0.11 & 0 & 0.1 & 0 & 0.14 & 0 \\
\hline & & \multicolumn{6}{|c|}{$\mathrm{R}^{2}$ of the fit with Lambert-Beer equation } \\
\hline \multirow{3}{*}{ nutrient limited } & 14 & \multicolumn{2}{|c|}{0.82} & \multicolumn{2}{|c|}{0.7} & \multicolumn{2}{|c|}{0.74} \\
\hline & 28 & \multicolumn{2}{|c|}{0.88} & \multicolumn{2}{|c|}{0.83} & \multicolumn{2}{|c|}{0.87} \\
\hline & 92 & \multicolumn{2}{|c|}{0.98} & \multicolumn{2}{|c|}{0.96} & \multicolumn{2}{|c|}{0.95} \\
\hline \multirow{3}{*}{$\begin{array}{l}\text { nutrients in } \\
\text { excess }\end{array}$} & 39.5 & \multicolumn{2}{|c|}{0.98} & \multicolumn{2}{|c|}{0.98} & \multicolumn{2}{|c|}{0.86} \\
\hline & 79 & \multicolumn{2}{|c|}{0.93} & \multicolumn{2}{|c|}{0.97} & \multicolumn{2}{|c|}{0.96} \\
\hline & 158 & \multicolumn{2}{|c|}{0.94} & \multicolumn{2}{|c|}{0.96} & \multicolumn{2}{|c|}{0.99} \\
\hline & & \multicolumn{6}{|c|}{$\mathrm{R}^{2}$ of the fit with Schuster's law } \\
\hline \multirow{3}{*}{ nutrient limited } & 14 & \multicolumn{2}{|c|}{0.96} & \multicolumn{2}{|c|}{0.97} & \multicolumn{2}{|c|}{0.98} \\
\hline & 28 & \multicolumn{2}{|c|}{0.99} & 0.9 & 98 & 0.5 & \\
\hline & 92 & 0. & 98 & 0.9 & 99 & 0. & \\
\hline & 39.5 & 0. & 98 & 0.5 & 98 & 0.5 & \\
\hline $\begin{array}{l}\text { nutrients in } \\
\text { excess }\end{array}$ & 79 & 0. & 93 & 0.9 & 97 & 0.9 & \\
\hline & 158 & 0. & 94 & 0.9 & 96 & 0.9 & \\
\hline
\end{tabular}


Table S6: Light parameters $a$ and $b$ estimated for Batch 1 defining the attenuation coefficient based on the TSS $\left(k_{a}=a * e^{-b * X_{A l g}}\right)$. Light parameters $c$ and $d$ estimated for Batch 1 defining the attenuation coefficient based on the chlorophyll content $\left(k_{a, p}=\frac{d}{X_{C h l}}-c\right)$.

\begin{tabular}{|c|c|c|c|c|}
\cline { 2 - 5 } \multicolumn{1}{c|}{} & $\begin{array}{c}\mathrm{a} \\
\left(\mathrm{m}^{2} / \mathrm{g} \mathrm{TSS}\right)\end{array}$ & $\begin{array}{c}\mathrm{b} \\
\left(\mathrm{m}^{3} / \mathrm{g} \mathrm{TSS}\right)\end{array}$ & $\begin{array}{c}\mathrm{c} \\
\left(\mathrm{m}^{2} / \mathrm{g} \mathrm{Chl}\right)\end{array}$ & $\begin{array}{c}\mathrm{d} \\
\left(\mathrm{m}^{3} / \mathrm{g} \text { Chl }\right)\end{array}$ \\
\hline Batch 1 & $0.135 \pm 0.009$ & $0.0018 \pm 0.0003$ & $1.06 \pm 0.8$ & $29.3 \pm 0.65$ \\
\hline
\end{tabular}

Table S7: RMSNE, AIC and ARILC values obtained with simulations using three different light modelling complexities, first using TSS to calculate the attenuation coefficient $\left(\mathrm{k}_{\mathrm{a}}\right)$ and second using the chlorophyll content to calculate the pigment specific attenuation coefficient $\left(\mathrm{k}_{\mathrm{a}, \mathrm{p}}\right)$.

\begin{tabular}{|l|c|c|c|c|c|c|}
\hline & \multicolumn{2}{|c|}{ calculated with ka } & \multicolumn{3}{c|}{ calculated with ka,p } \\
\hline & $\begin{array}{c}\text { Average } \\
\text { light }\end{array}$ & $\begin{array}{c}\text { Variable } \\
\text { average } \\
\text { light }\end{array}$ & $\begin{array}{c}\text { Layer } \\
\text { model }\end{array}$ & $\begin{array}{c}\text { Average } \\
\text { light }\end{array}$ & $\begin{array}{c}\text { Variable } \\
\text { average } \\
\text { light }\end{array}$ & $\begin{array}{c}\text { Layer } \\
\text { model }\end{array}$ \\
\hline \multicolumn{7}{|c|}{ RMSNE (-) } \\
\hline $\mathrm{X}_{\mathrm{Alg}}$ & 0.167 & 0.157 & 0.092 & 0.149 & 0.159 & 0.094 \\
\hline $\mathrm{X}_{\mathrm{AlgN}}$ & 0.124 & 0.142 & 0.171 & 0.125 & 0.149 & 0.164 \\
\hline $\mathrm{S}_{\mathrm{NO} 3}$ & 0.88 & 0.87 & 0.889 & 0.879 & 0.881 & 0.879 \\
\hline $\mathrm{X}_{\mathrm{AlgP}}$ & 0.281 & 0.284 & 0.229 & 0.277 & 0.24 & 0.293 \\
\hline $\mathrm{S}_{\mathrm{PO} 4}$ & 0.803 & 0.885 & 1.036 & 0.775 & 1.379 & 0.692 \\
\hline sum & 2.255 & 2.338 & 2.417 & 2.204 & 2.808 & 2.121 \\
\hline \multicolumn{7}{|c|}{ AIC (-) } \\
\hline $\mathrm{X}_{\mathrm{Alg}}$ & -97 & -101 & -134 & -104 & -100 & -133 \\
\hline $\mathrm{X}_{\mathrm{AlgN}}$ & -115 & -107 & -96 & -115 & -104 & -98 \\
\hline $\mathrm{S}_{\mathrm{NO} 3}$ & 6 & 5 & 7 & 6 & 6 & 6 \\
\hline $\mathrm{X}_{\mathrm{AlgP}}$ & -65 & -64 & -77 & -66 & -75 & -62 \\
\hline $\mathrm{S}_{\mathrm{PO} 4}$ & 0.4 & 6 & 16 & -2 & 33.9 & -9 \\
\hline sum & -271 & -260 & -284 & -281 & -239 & -296 \\
\hline \multicolumn{7}{|c|}{ ARILC (-) } \\
\hline $\mathrm{X}_{\mathrm{Alg}}$ & 0.0043 & 0.0041 & 0.0011 & 0.0041 & 0.0039 & 0.0036 \\
\hline $\mathrm{X}_{\mathrm{AlgN}}$ & 0.003 & 0.0031 & 0.0007 & 0.0033 & 0.0033 & 0.0048 \\
\hline $\mathrm{S}_{\mathrm{NO} 3}$ & 0.033 & 0.022 & 0.0074 & 0.026 & 0.02 & 0.018 \\
\hline $\mathrm{X}_{\mathrm{AlgP}}$ & 0.0081 & 0.0077 & 0.0032 & 0.0091 & 0.0071 & 0.0084 \\
\hline $\mathrm{S}_{\mathrm{PO} 4}$ & 0.023 & 0.021 & 0.02 & 0.022 & 0.034 & 0.019 \\
\hline sum & 0.071 & 0.057 & 0.033 & 0.065 & 0.068 & 0.054 \\
\hline
\end{tabular}


Table S8: Comparison of the estimated parameter subsets using the three complexity levels. The values are presented as mean $\pm 95 \%$ confidence interval.

\begin{tabular}{|l|c|c|c|c|c|c|}
\cline { 2 - 7 } \multicolumn{1}{c|}{} & \multicolumn{3}{c|}{ calculated with ka } & \multicolumn{3}{c|}{ calculated with ka,p } \\
\cline { 2 - 7 } & $\begin{array}{c}\text { CL-1 } \\
\text { Average } \\
\text { light }\end{array}$ & $\begin{array}{c}\text { CL-2 } \\
\text { Variable } \\
\text { average } \\
\text { light }\end{array}$ & $\begin{array}{c}\text { CL-3 } \\
\text { Layer model }\end{array}$ & $\begin{array}{c}\text { CL-1 } \\
\text { Average } \\
\text { light }\end{array}$ & $\begin{array}{c}\text { CL-2 } \\
\text { Variable } \\
\text { average } \\
\text { light }\end{array}$ & $\begin{array}{c}\text { CL-3 } \\
\text { Layer model }\end{array}$ \\
\hline$\mu_{A, \max }\left(\mathrm{d}^{-1}\right)$ & $4.1 \pm 1.91$ & $3.81 \pm 1.51$ & $6.2 \pm 0.53$ & $4.08 \pm 1.69$ & $5.28 \pm 1.8$ & $5.5 \pm 1.27$ \\
\hline$K_{N O, A l g}\left(\mathrm{gN} \mathrm{m}^{-3}\right)$ & $14.83 \pm 0.56$ & $14.59 \pm 1.53$ & $14.61 \pm 1.46$ & $14.86 \pm 0.4$ & $14.52 \pm 1.68$ & $14.82 \pm 0.42$ \\
\hline$K_{P O 4, A l g}\left(\mathrm{gP} \mathrm{m}^{-3}\right)$ & $4.22 \pm 1.38$ & $4.19 \pm 1.41$ & $4.31 \pm 1.52$ & $4.14 \pm 1.42$ & $4.49 \pm 1.37$ & $4.07 \pm 1.52$ \\
\hline$k_{N O, A l g}\left(\mathrm{gN} \mathrm{g}^{-1} \mathrm{COD} \mathrm{d}^{-1}\right)$ & $0.13 \pm 0.068$ & $0.1 \pm 0.034$ & $0.17 \pm 0.025$ & $0.13 \pm 0.067$ & $0.12 \pm 0.044$ & $0.16 \pm 0.04$ \\
\hline$k_{P O 4, A l g}\left(\mathrm{gN} \mathrm{g}^{-1} \mathrm{COD} \mathrm{d}^{-1}\right)$ & $0.016 \pm 0.006$ & $0.015 \pm 0.005$ & $0.014 \pm 0.004$ & $0.016 \pm 0.006$ & $0.011 \pm 0.003$ & $0.018 \pm 0.006$ \\
\hline$b_{A l g}\left(\mathrm{~d}^{-1}\right)$ & $0.24 \pm 0.025$ & $0.24 \pm 0.024$ & $0.25 \pm 0.008$ & $0.24 \pm 0.024$ & $0.25 \pm 0.006$ & $0.22 \pm 0.036$ \\
\hline
\end{tabular}

Table S9: Model calibration and identifiability analysis using the average constant light intensityCL1. TSS was used to calculate the attenuation coefficient $\left(\mathrm{k}_{\mathrm{a}}\right)$. Histograms obtained for the posterior parameter distribution and correlation matrix.

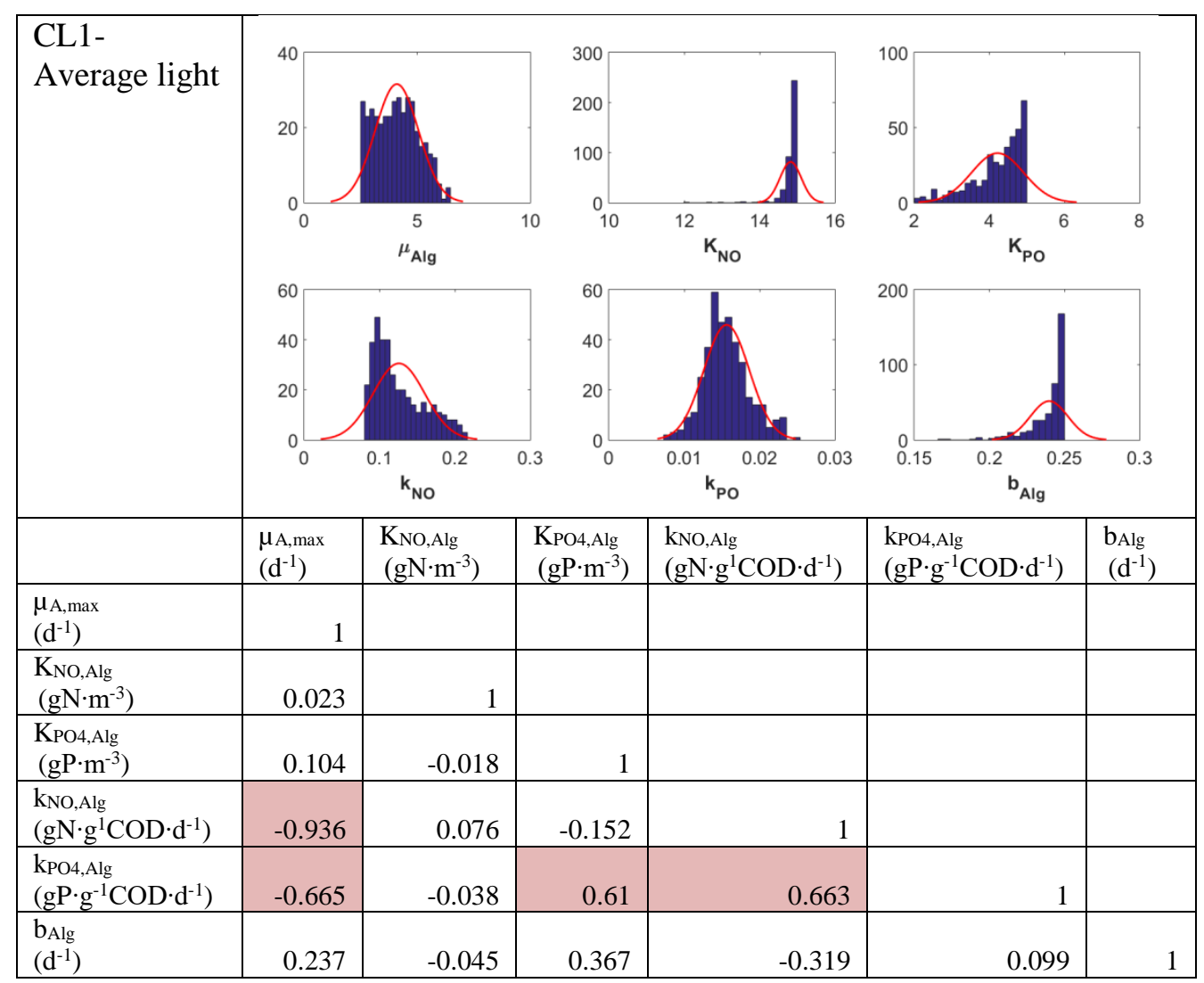


Table S10: Model calibration and identifiability analysis using the average constant light intensity CL1. The chlorophyll content was used to calculate the pigment specific attenuation coefficient $\left(\mathrm{k}_{\mathrm{a}, \mathrm{p}}\right)$. Histograms obtained for the posterior parameter distribution and correlation matrix.

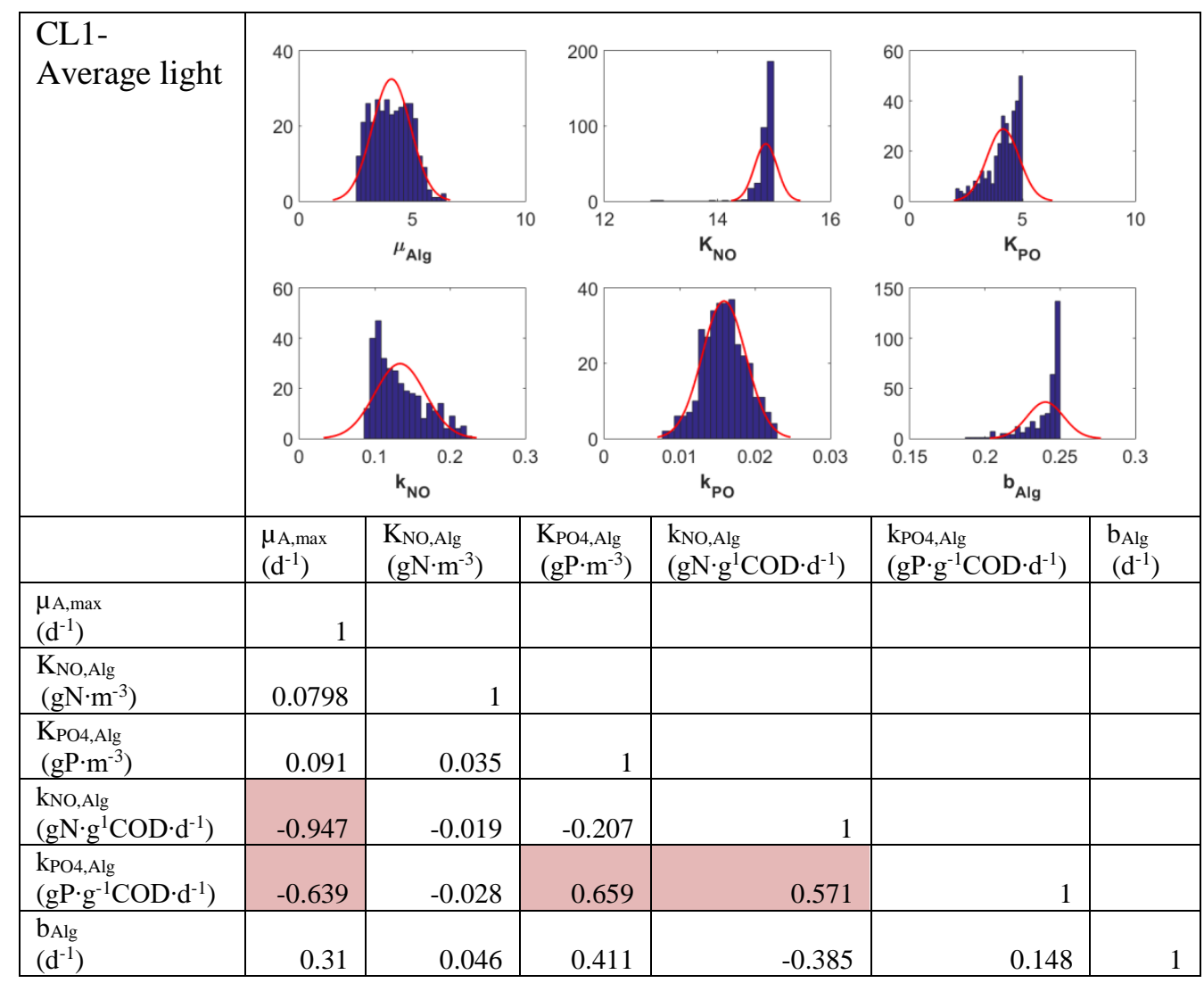


Table S11: Model calibration and identifiability analysis using the variable average light intensity CL2. TSS was used to calculate the attenuation coefficient $\left(k_{a}\right)$. Histograms obtained for the posterior parameter distribution and correlation matrix.

\begin{tabular}{|l|l|l|l|l|l|l|l|l|}
\hline $\begin{array}{l}\text { CL2- } \\
\text { Variable } \\
\text { average light }\end{array}$ & & \\
\end{tabular}


Table S12: Model calibration and identifiability analysis using the variable average light intensity CL2. The chlorophyll content was used to calculate the pigment specific attenuation coefficient $\left(\mathrm{k}_{\mathrm{a}, \mathrm{p}}\right)$. Histograms obtained for the posterior parameter distribution and correlation matrix.

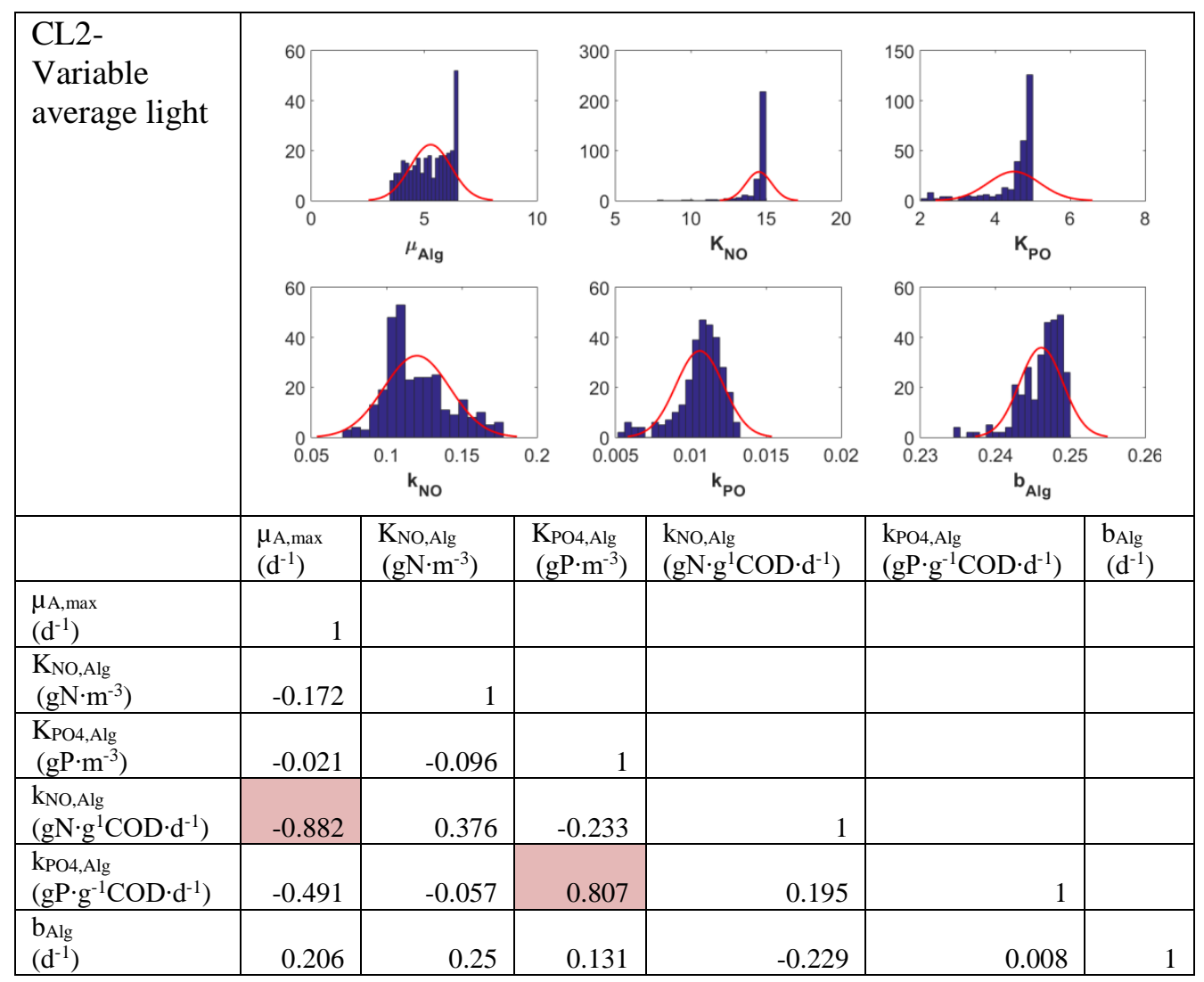


Table S13: Model calibration and identifiability analysis using the layer model - CL3. TSS was used to calculate the attenuation coefficient $\left(\mathrm{k}_{\mathrm{a}}\right)$. Histograms obtained for the posterior parameter distribution and correlation matrix.

\begin{tabular}{|c|c|c|c|c|c|c|}
\hline $\begin{array}{l}\text { CL3- Layer } \\
\text { model }\end{array}$ & 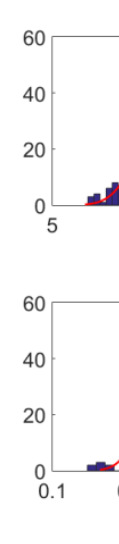 & ${ }_{\mathbf{k}_{\text {No }}}^{0.2}$ & 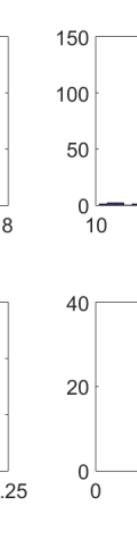 & 20 & (2) & $\underbrace{}_{0.26}$ \\
\hline & $\begin{array}{l}\mu_{\mathrm{A}, \max } \\
\left(\mathrm{d}^{-1}\right)\end{array}$ & $\begin{array}{l}\begin{array}{l}\mathrm{K}_{\mathrm{NO}, \mathrm{Alg}} \\
\left(\mathrm{gN} \cdot \mathrm{m}^{-3}\right)\end{array} \\
\end{array}$ & $\begin{array}{l}\begin{array}{l}\text { KPO4,Alg } \\
\left(\mathrm{gP} \cdot \mathrm{m}^{-3}\right)\end{array} \\
\end{array}$ & $\begin{array}{l}\text { kNO,Alg } \\
\left(\mathrm{gN} \cdot \mathrm{g}^{1} \mathrm{COD} \cdot \mathrm{d}^{-1}\right)\end{array}$ & $\begin{array}{l}\text { kPO4,Alg } \\
\left(\mathrm{gP} \cdot \mathrm{g}^{-1} \mathrm{COD} \cdot \mathrm{d}^{-1}\right)\end{array}$ & $\begin{array}{l}\mathrm{b}_{\mathrm{Alg}} \\
\left(\mathrm{d}^{-1}\right)\end{array}$ \\
\hline $\begin{array}{l}\mu_{\mathrm{A}, \max } \\
\left(\mathrm{d}^{-1}\right)\end{array}$ & 1 & & & & & \\
\hline $\begin{array}{l}\mathrm{K}_{\mathrm{NO}, \mathrm{Alg}} \\
\left(\mathrm{gN} \cdot \mathrm{m}^{-3}\right)\end{array}$ & 0.005 & 1 & & & & \\
\hline $\begin{array}{l}\mathrm{KPO} 4, \mathrm{Alg} \\
\left(\mathrm{gP} \cdot \mathrm{m}^{-3}\right)\end{array}$ & -0.116 & -0.003 & 1 & & & \\
\hline $\begin{array}{l}\mathrm{kNO}, \mathrm{Alg} \\
\left(\mathrm{gN} \cdot \mathrm{g}^{1} \mathrm{COD} \cdot \mathrm{d}^{-1}\right)\end{array}$ & -0.213 & 0.596 & -0.423 & 1 & & \\
\hline $\begin{array}{l}\mathrm{kPO} 4, \mathrm{Alg} \\
\left(\mathrm{gP} \cdot \mathrm{g}^{-1} \mathrm{COD} \cdot \mathrm{d}^{-1}\right)\end{array}$ & -0.293 & -0.026 & 0.931 & -0.518 & 1 & \\
\hline $\begin{array}{l}b_{\text {Alg }} \\
\left(d^{-1}\right)\end{array}$ & 0.186 & 0.195 & -0.006 & 0.034 & 0.041 & \\
\hline
\end{tabular}


Table S14: Model calibration and identifiability analysis using the layer model - CL3. The chlorophyll content was used to calculate the pigment specific attenuation coefficient $\left(\mathrm{k}_{\mathrm{a}, \mathrm{p}}\right)$. Histograms obtained for the posterior parameter distribution and correlation matrix.

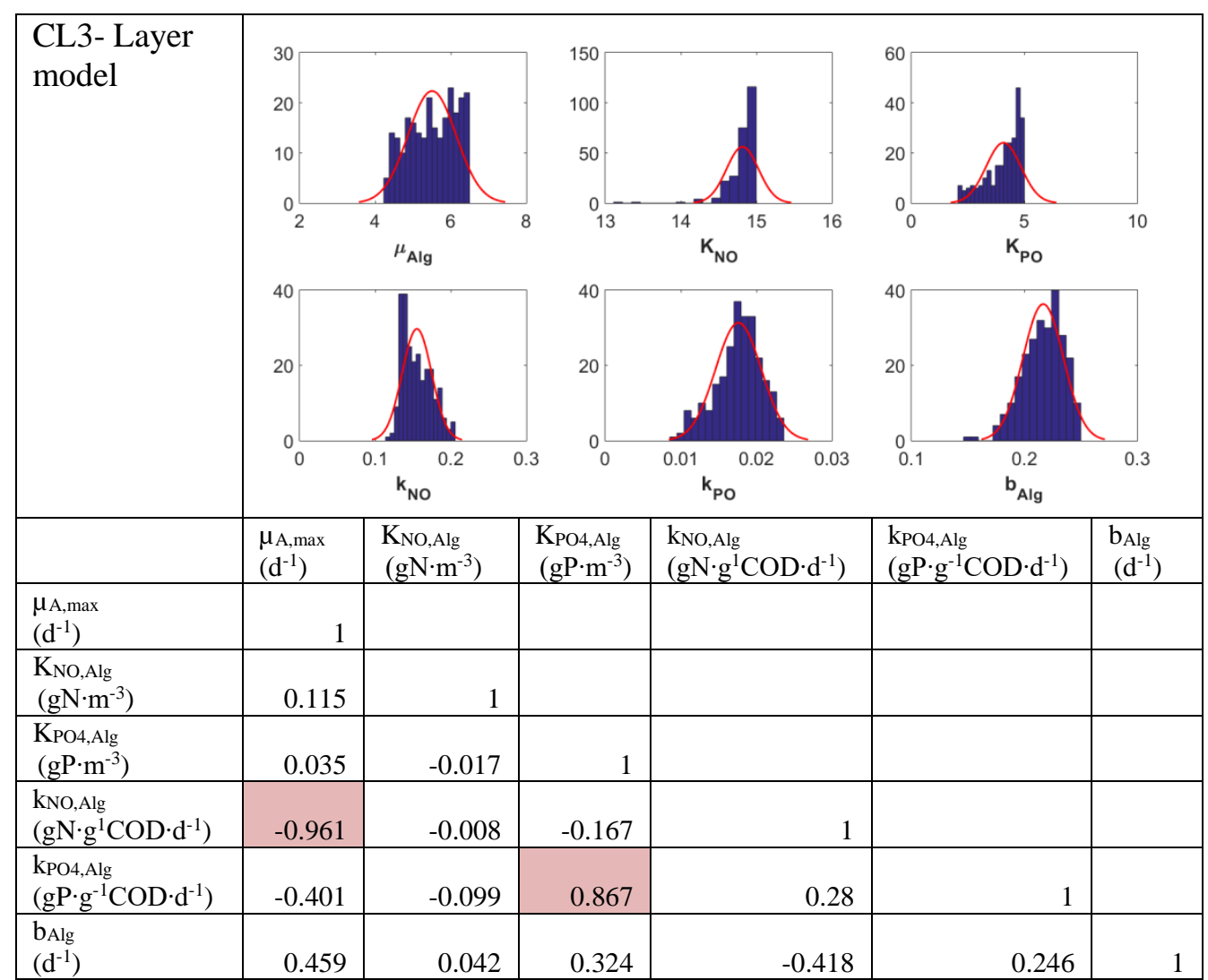


$\underline{\text { Figures }}$

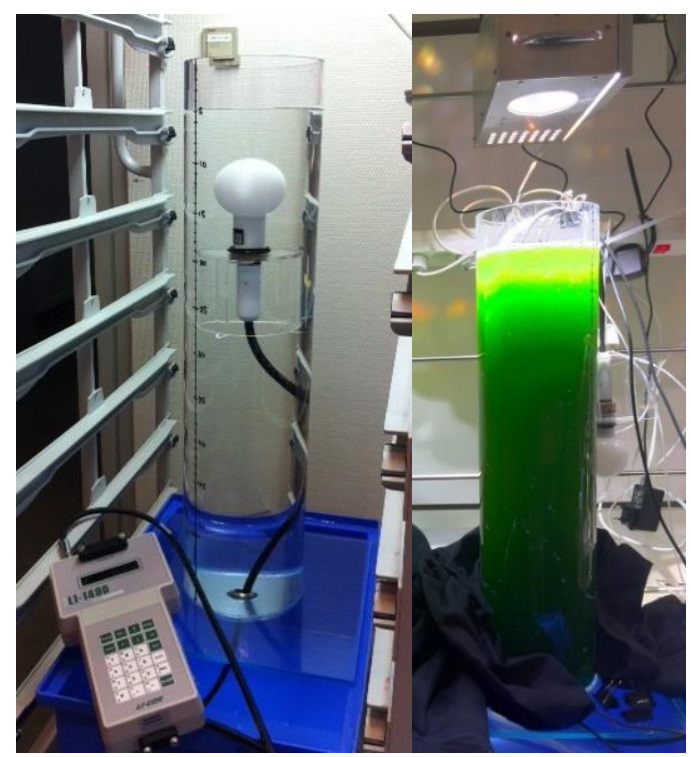

Figure S1: The cylindrical shaped clear walled plastic reactor used for the experiments, with the light sensor inside, connected to a data logger (picture on the left). The 8-L reactor used for the batch experiments with the custom built lamp mounted above (picture on the right). The black cloth on the bottom was used to cover the reactor wall from the side.

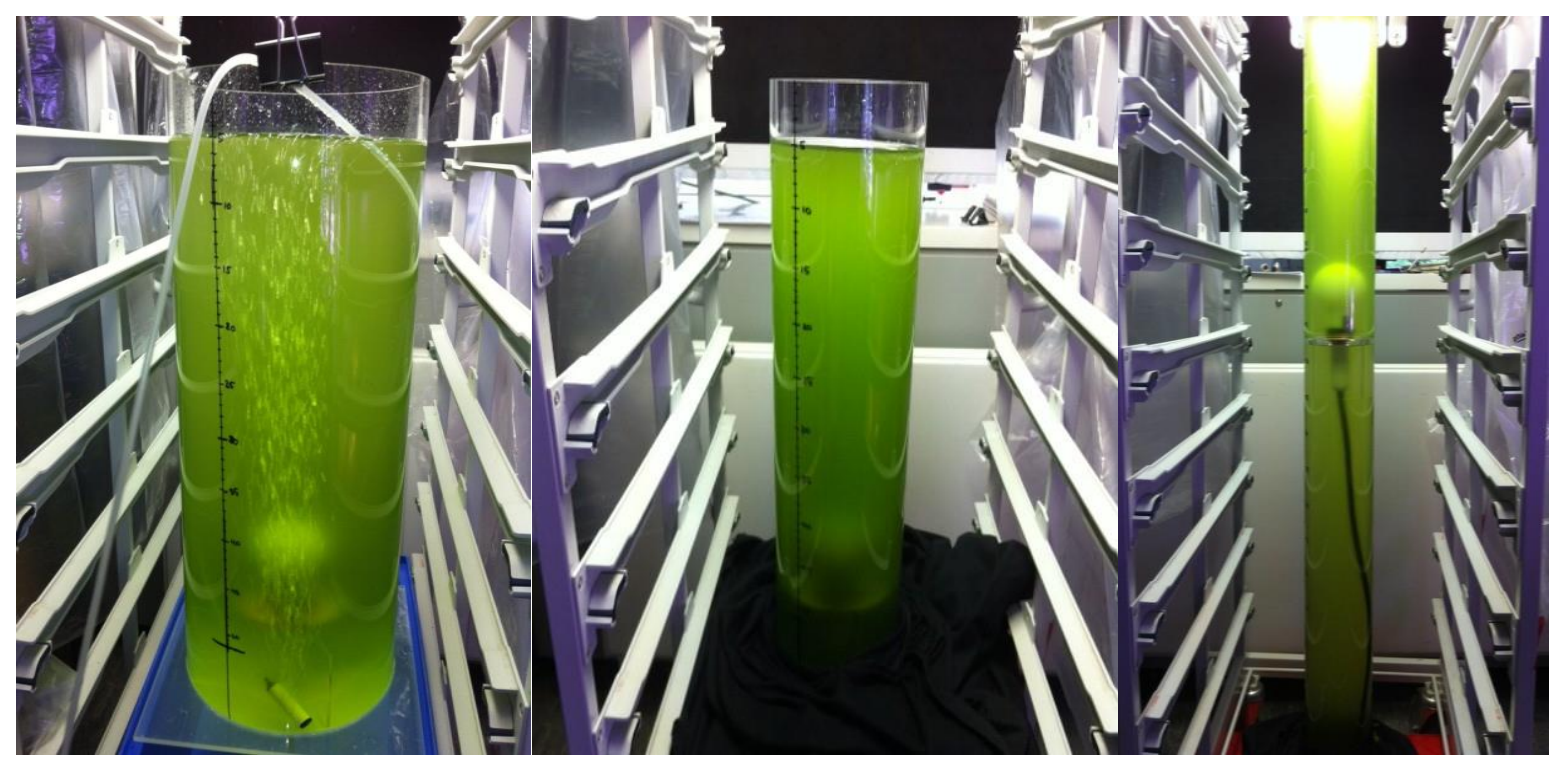

Figure S2: The cylindrical shaped clear walled plastic reactors with three different diameters used for the experiments. Reactor 1 with a diameter of $240 \mathrm{~mm}$ (picture on the left). Reactor 2 with a diameter of $140 \mathrm{~mm}$ (picture in the middle). Reactor 3 with a diameter of $110 \mathrm{~mm}$ (picture on the right). 


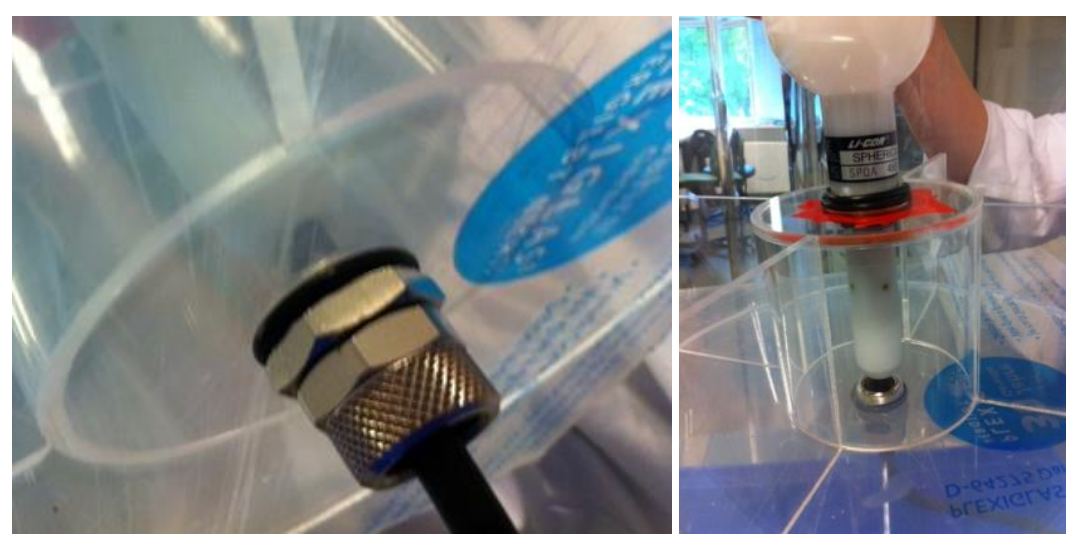

Figure S3: The fitting on the bottom of the reactor, used to mount the cable of the light sensor (picture on the left). The plastic fitting, used to keep the light sensor in a vertical upward position inside the reactor (picture on the right).
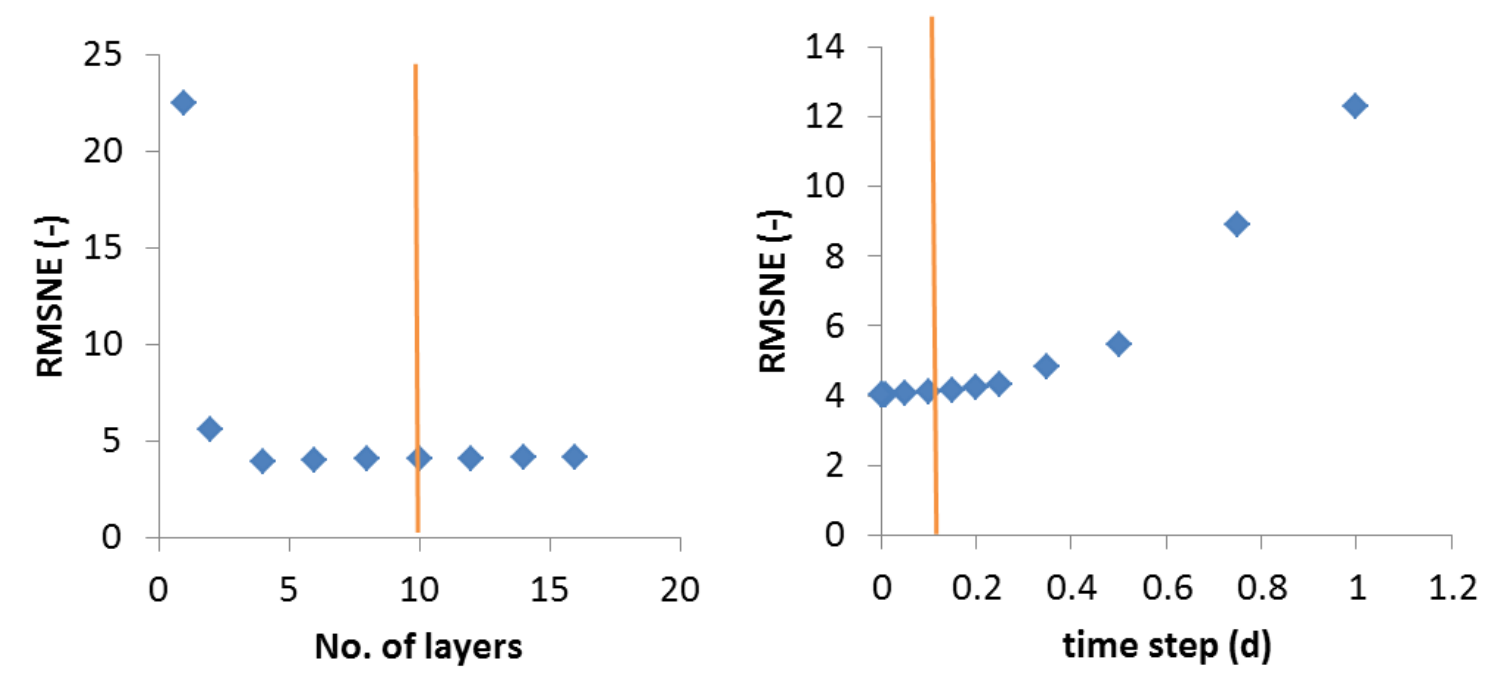

Figure S4: Evaluation of optimal number of layers and optimal time-step of the layer model based on the RMSNE of the simulation. The RMSNE was calculated by comparing the simulation to the experimental data in Batch 1. 


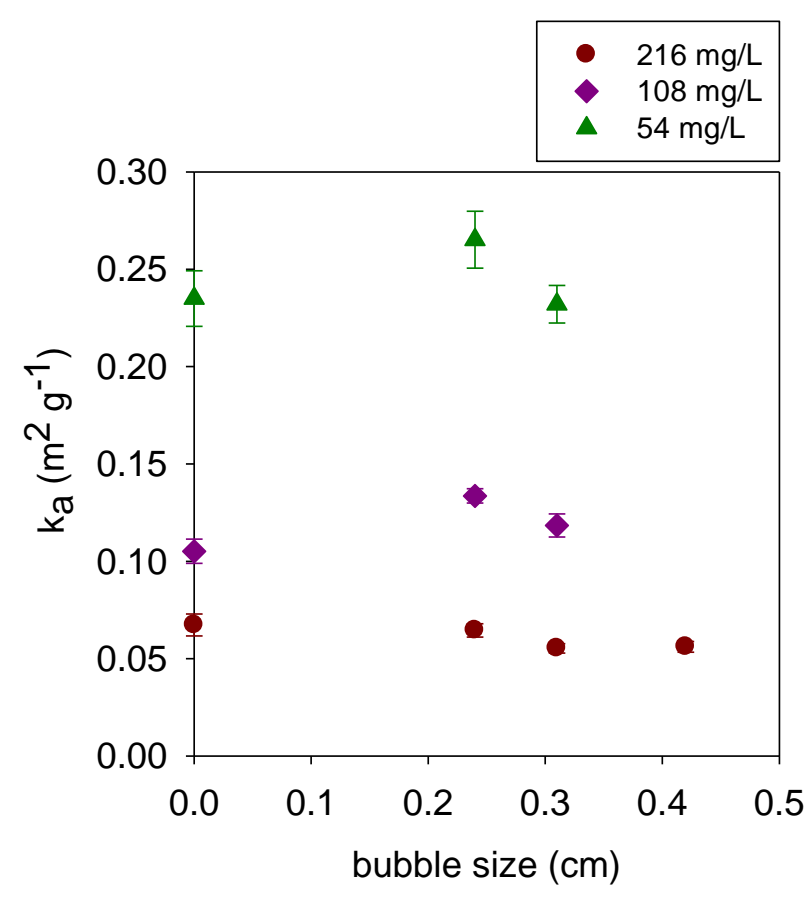

Figure S5: Light attenuation inside the PBR with $140 \mathrm{~mm}$ diameter assessing the effect of bubble size.

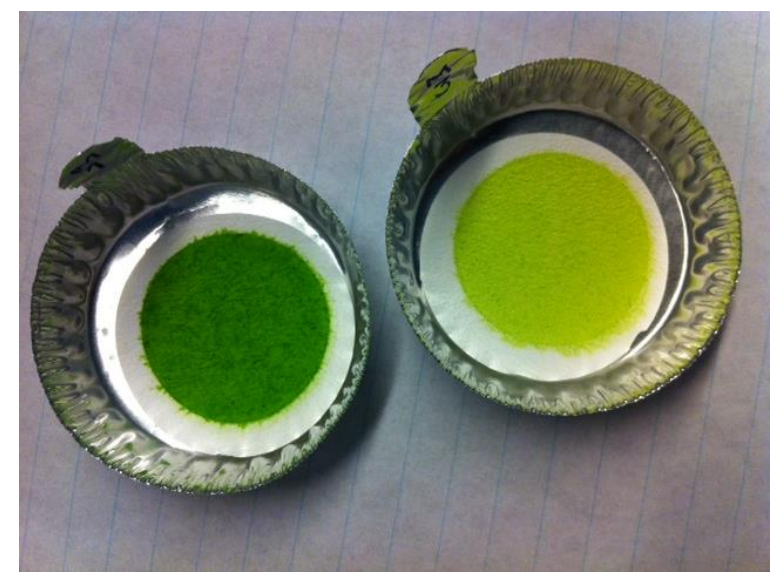

Figure S6: Colour change due to nutrient limited conditions (see Fig. 2a). The left metal plate contains a glass-fibre filter that has a deep green colour, due to high chlorophyll content at nutrients in excess conditions (see Fig. 2b). The metal plate on the right contains a filter that has yellowish colour due to the increase in carotenoid level under nutrient limited conditions. 

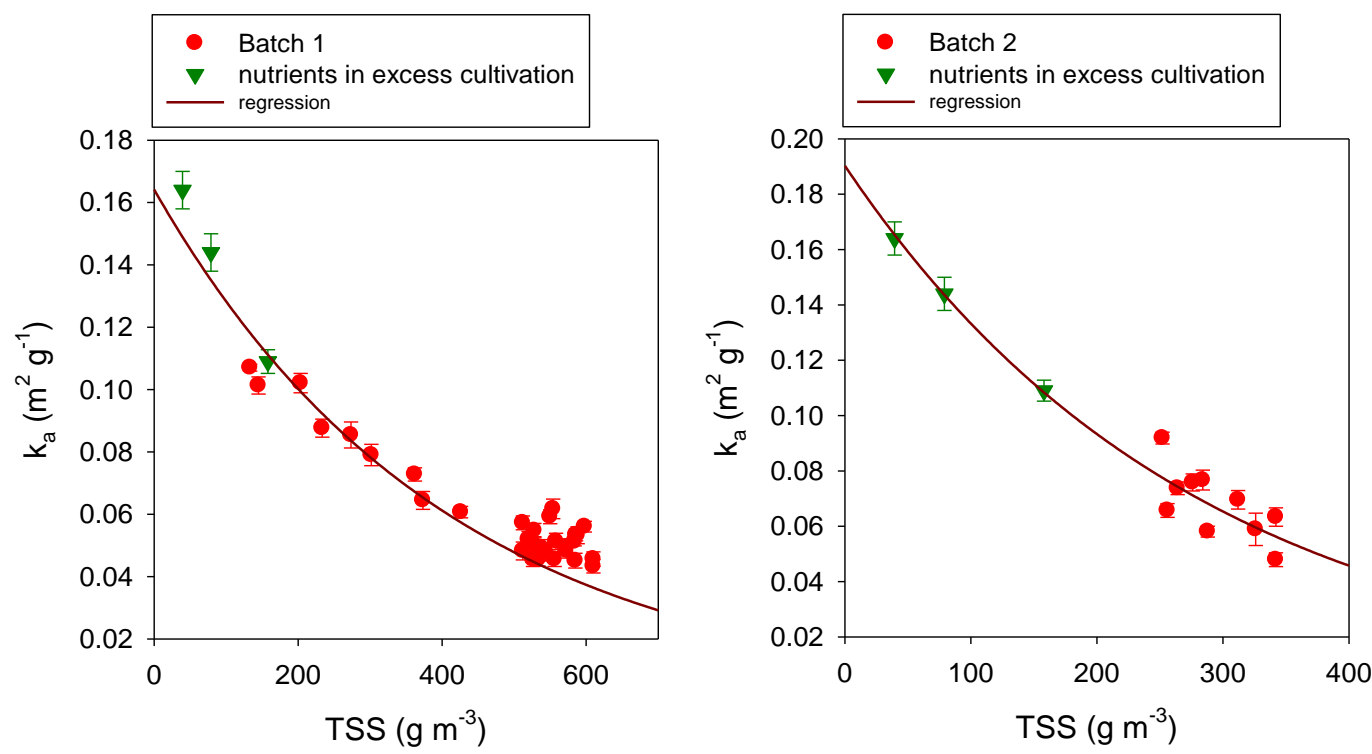

Figure S7: Attenuation coefficient during the batch cultivation as a function of biomass concentration. The figures include the data for nutrients in excess cultivation, for comparison with the batches.
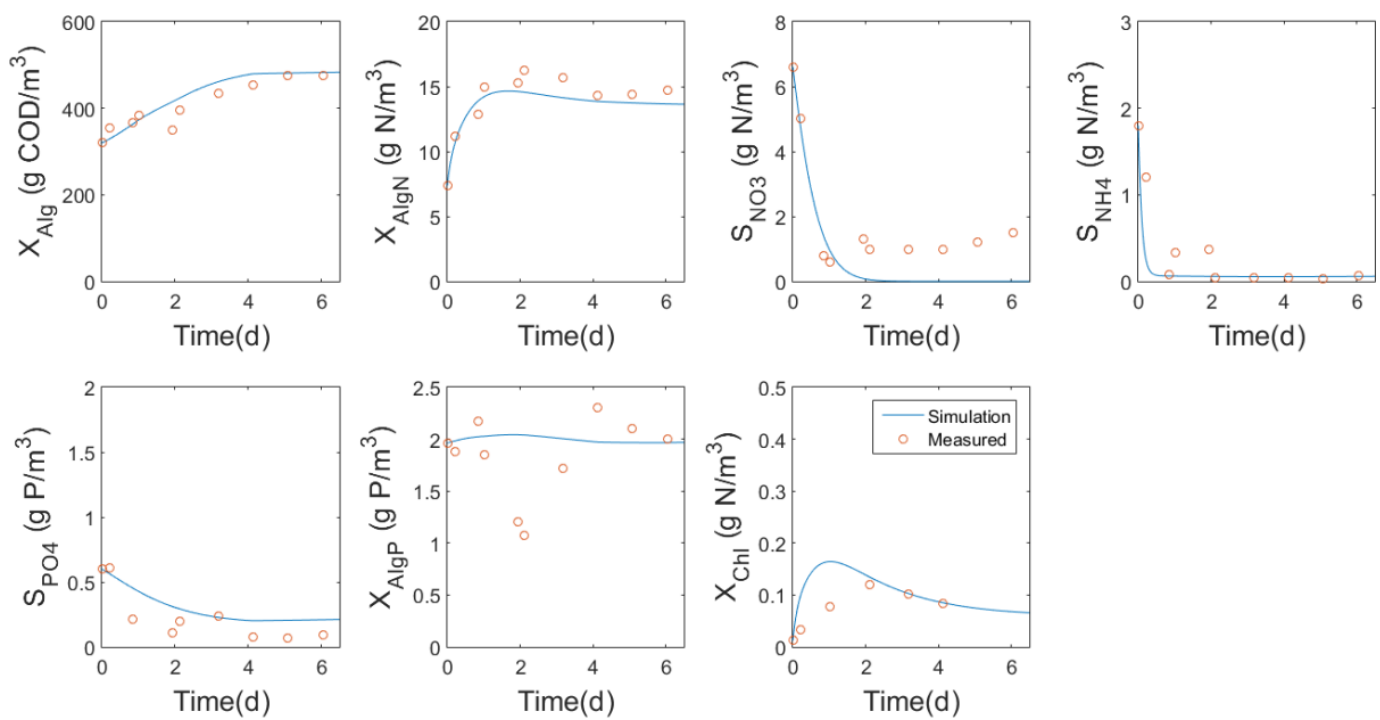

Figure S8: Simulations of Batch 2 using the parameter set estimated in Batch 1. 

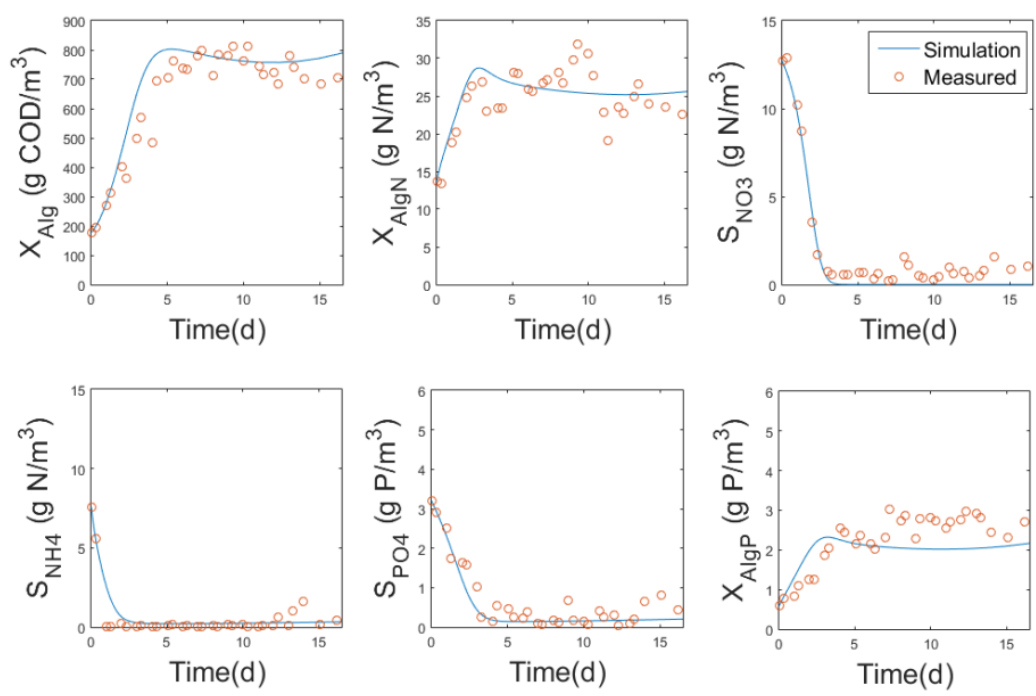

Figure S9: Simulation using model CL-1 (average constant light intensity, $127 \mu \mathrm{mol} \mathrm{m} \mathrm{m}^{-2} \mathrm{~s}^{-1}$ ) with the mean values of the paremeters estimated. TSS was used to calculate the attenuation coefficient $\left(\mathrm{k}_{\mathrm{a}}\right)$.
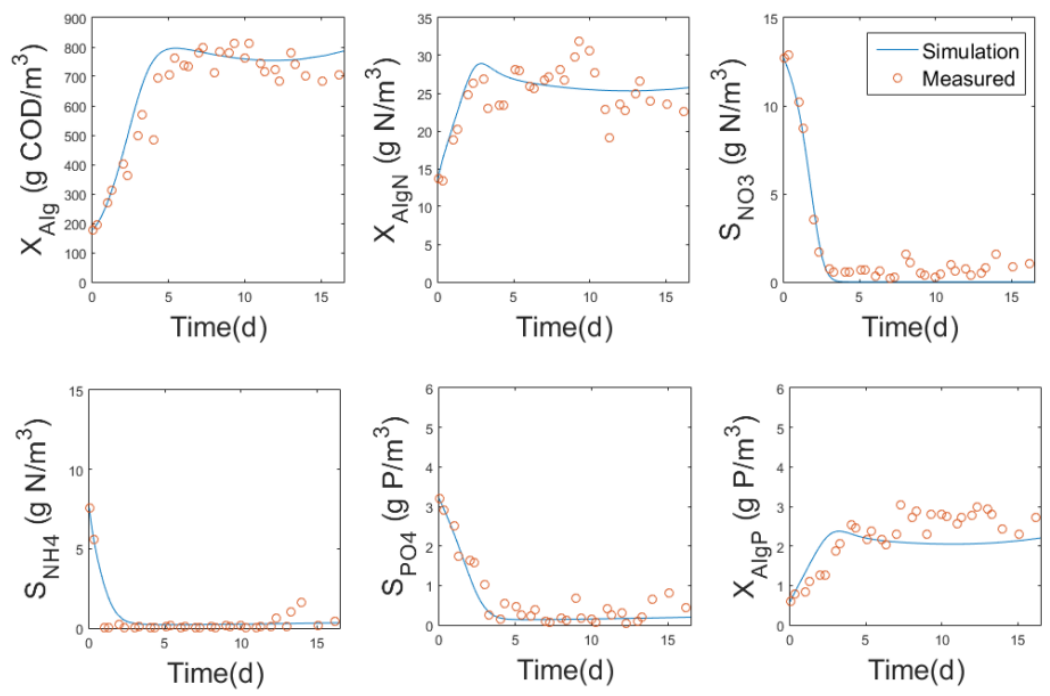

Figure S10: Simulation using model CL-1 (average constant light intensity, $118 \mu \mathrm{mol} \mathrm{m} \mathrm{m}^{-2} \mathrm{~s}^{-1}$ ) with the mean values of the paremeters estimated. The chlorophyll content was used to calculate the pigment specific attenuation coefficient $\left(\mathrm{k}_{\mathrm{a}, \mathrm{p}}\right)$. 

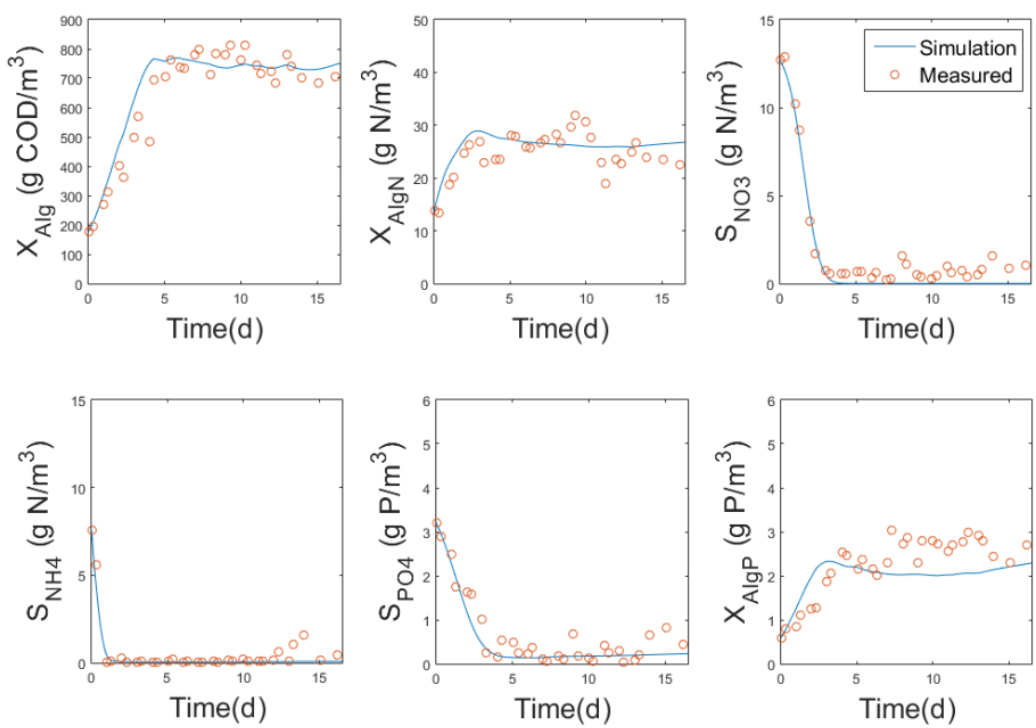

Figure S11: Simulation using model CL-2 (time-variable light intensity) with the mean values of the paremeters estimated. TSS was used to calculate the attenuation coefficient $\left(\mathrm{k}_{\mathrm{a}}\right)$.
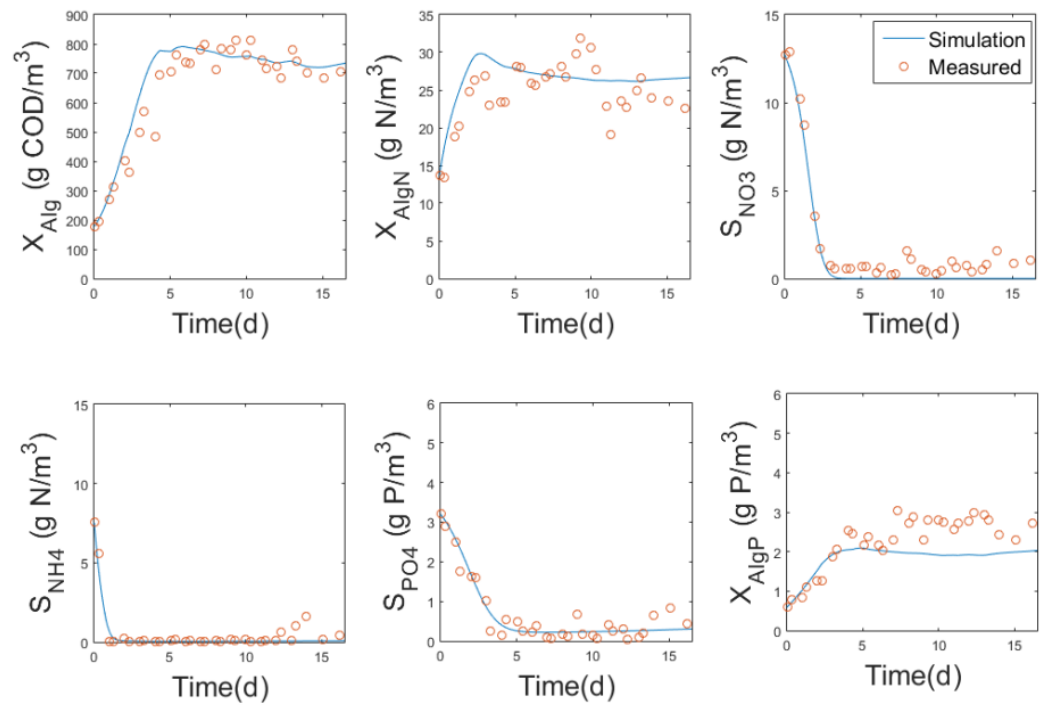

Figure S12: Simulation using model CL-2 (time-variable light intensity) with the mean values of the paremeters estimated. The chlorophyll content was used to calculate the pigment specific attenuation coefficient $\left(\mathrm{k}_{\mathrm{a}, \mathrm{p}}\right)$. 

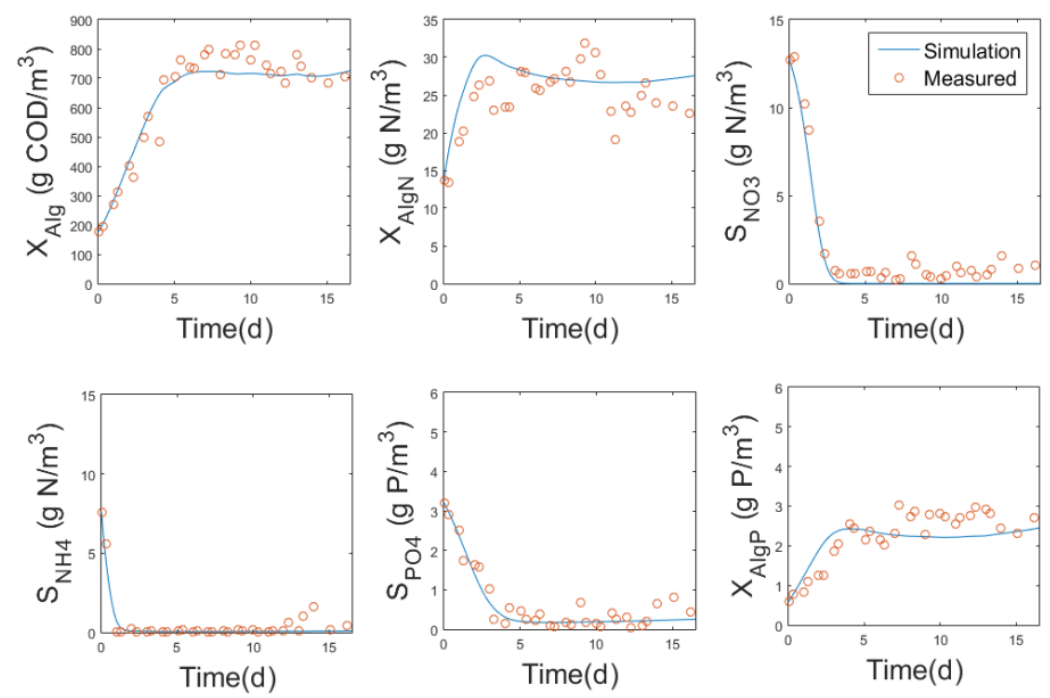

Figure S13: Simulation using model CL-3 (discretized layer model) with the mean values of the paremeters estimated. TSS was used to calculate the attenuation coefficient $\left(\mathrm{k}_{\mathrm{a}}\right)$.
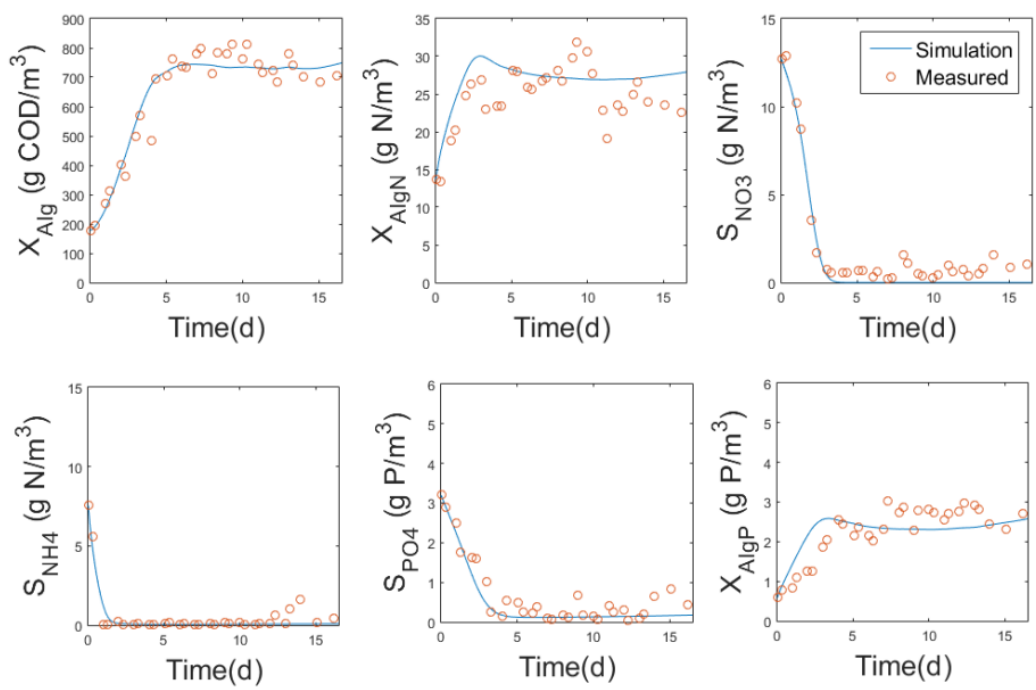

Figure S14: Simulation using model CL-3 (discretized layer model) with the mean values of the paremeters estimated. The chlorophyll content was used to calculate the pigment specific attenuation coefficient $\left(\mathrm{k}_{\mathrm{a}, \mathrm{p}}\right)$. 

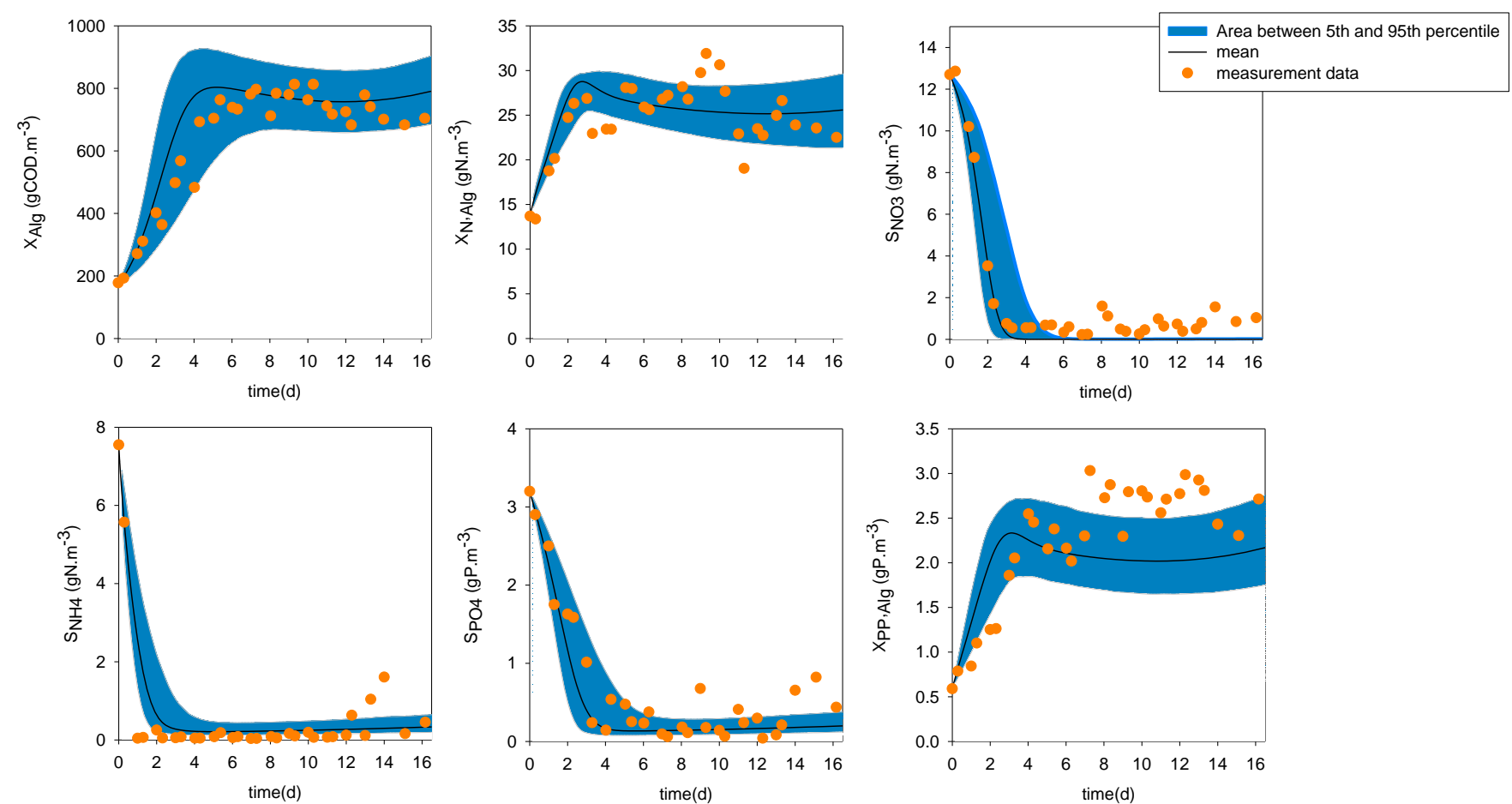

Figure S15: Simulation using model CL-1 (average constant light intensity, $127 \mu \mathrm{mol} \mathrm{m}^{-2} \mathrm{~s}^{-1}$ ) with the mean values of the parameters estimated. The uncertainty bands are shown in blue. The TSS is used to calculate the attenuation coefficient $\left(\mathrm{k}_{\mathrm{a}}\right)$. 

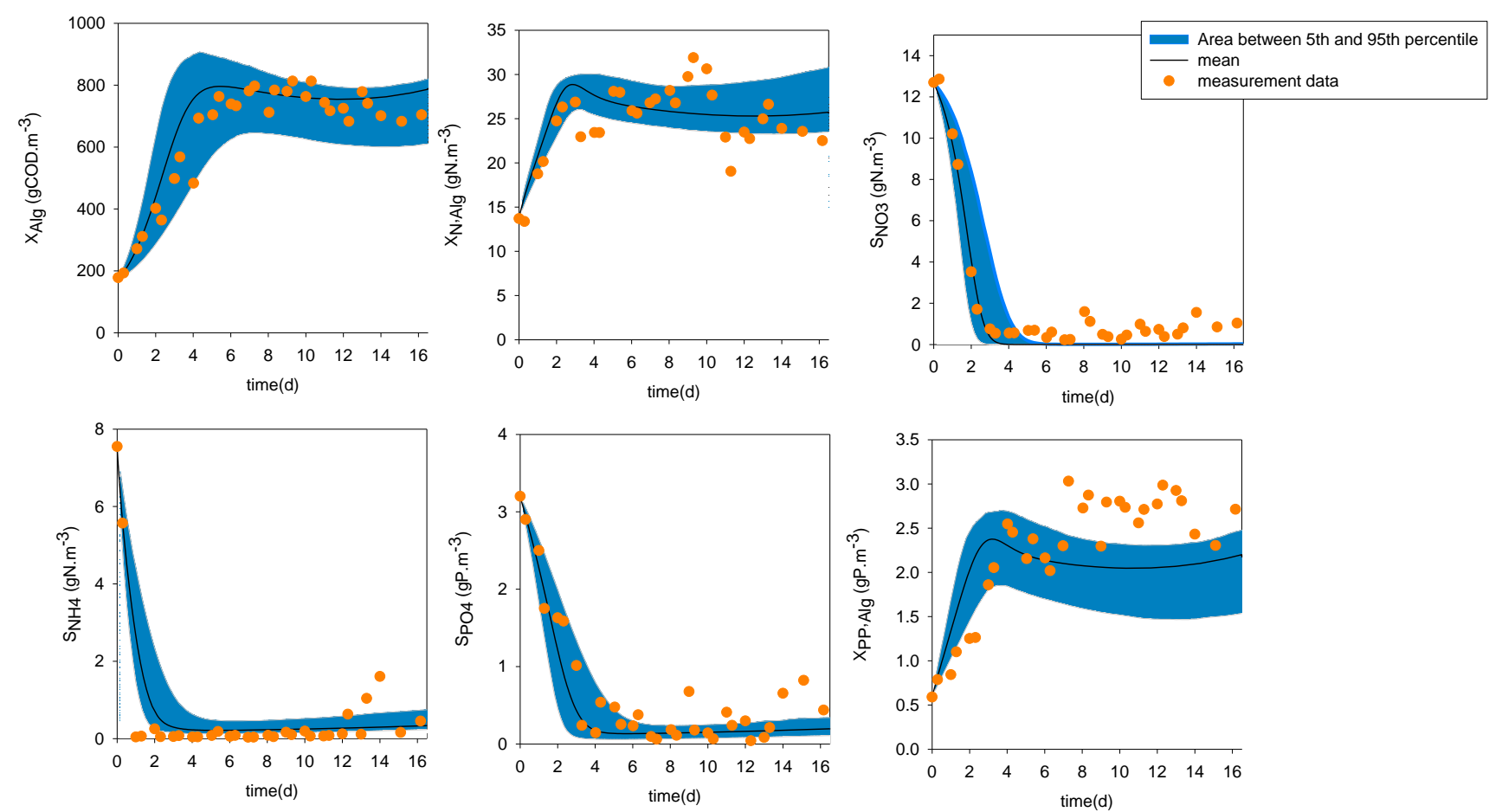

Figure S16: Simulation using model CL-1 (average constant light intensity, $118 \mu \mathrm{mol} \mathrm{m}^{-2} \mathrm{~s}^{-1}$ ) with the mean values of the parameters estimated. The uncertainty bands are shown in blue. The chlorophyll content is used to calculate the pigment specific attenuation coefficient $\left(\mathrm{k}_{\mathrm{a}, \mathrm{p}}\right)$. 

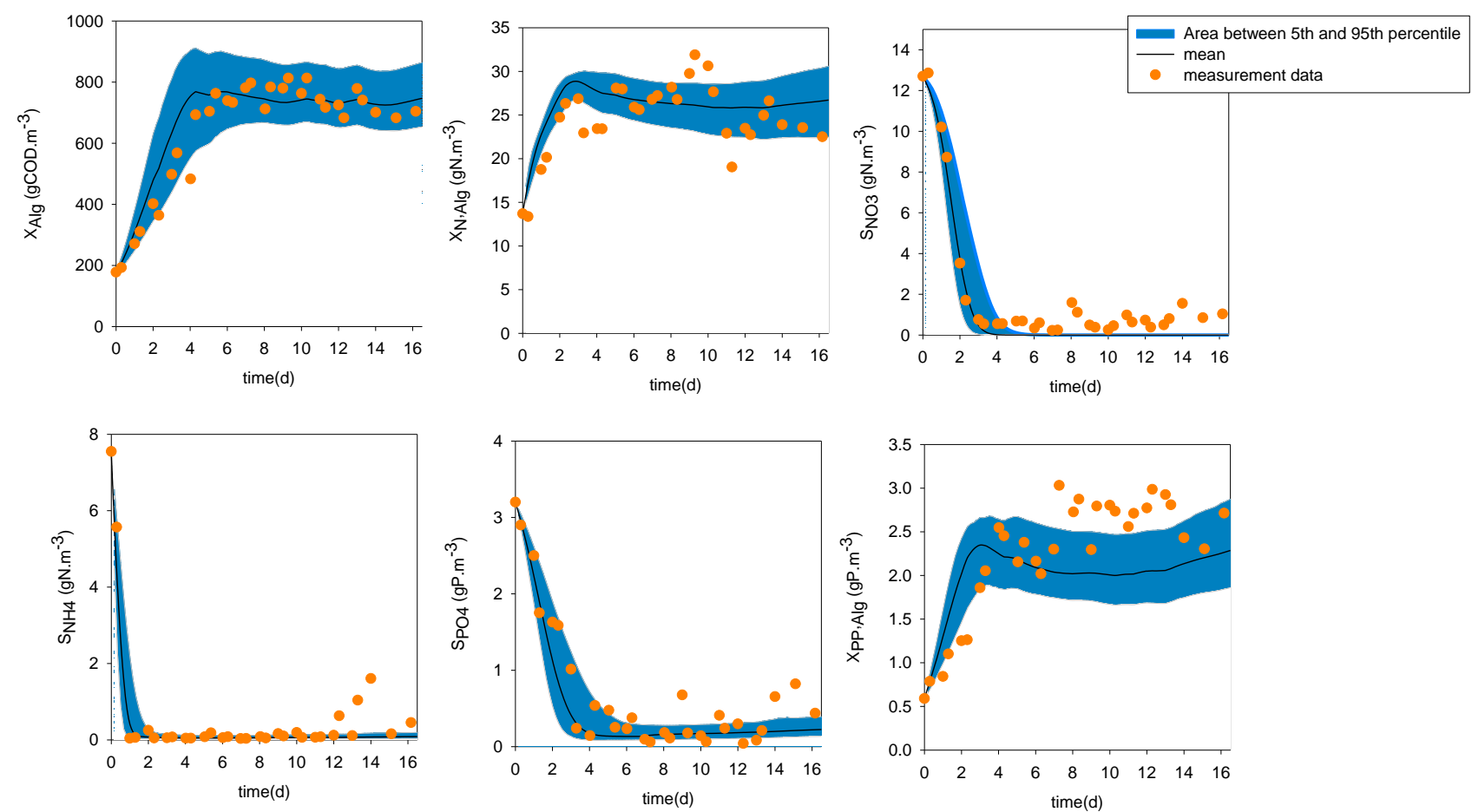

Figure S17: Simulation using model CL- 2 (time-variable light intensity) with the mean values of the parameters estimated. The uncertainty bands are shown in blue. The TSS is used to calculate the attenuation coefficient $\left(\mathrm{k}_{\mathrm{a}}\right)$. 

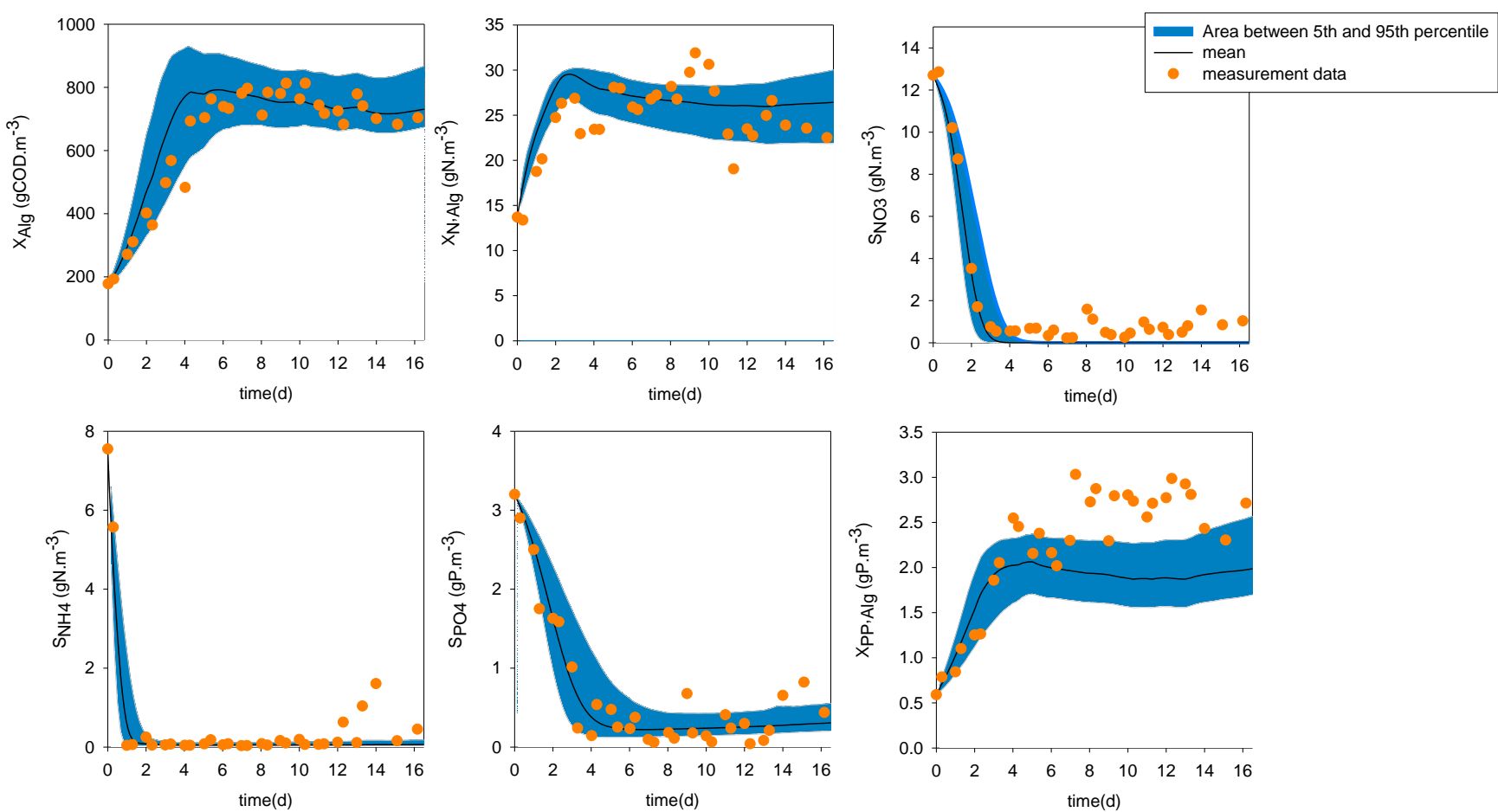

Figure S18: Simulation using model CL- 2 (time-variable light intensity) with the mean values of the parameters estimated. The uncertainty bands are shown in blue. The chlorophyll content is used to calculate the pigment specific attenuation coefficient $\left(\mathrm{k}_{\mathrm{a}, \mathrm{p}}\right)$. 

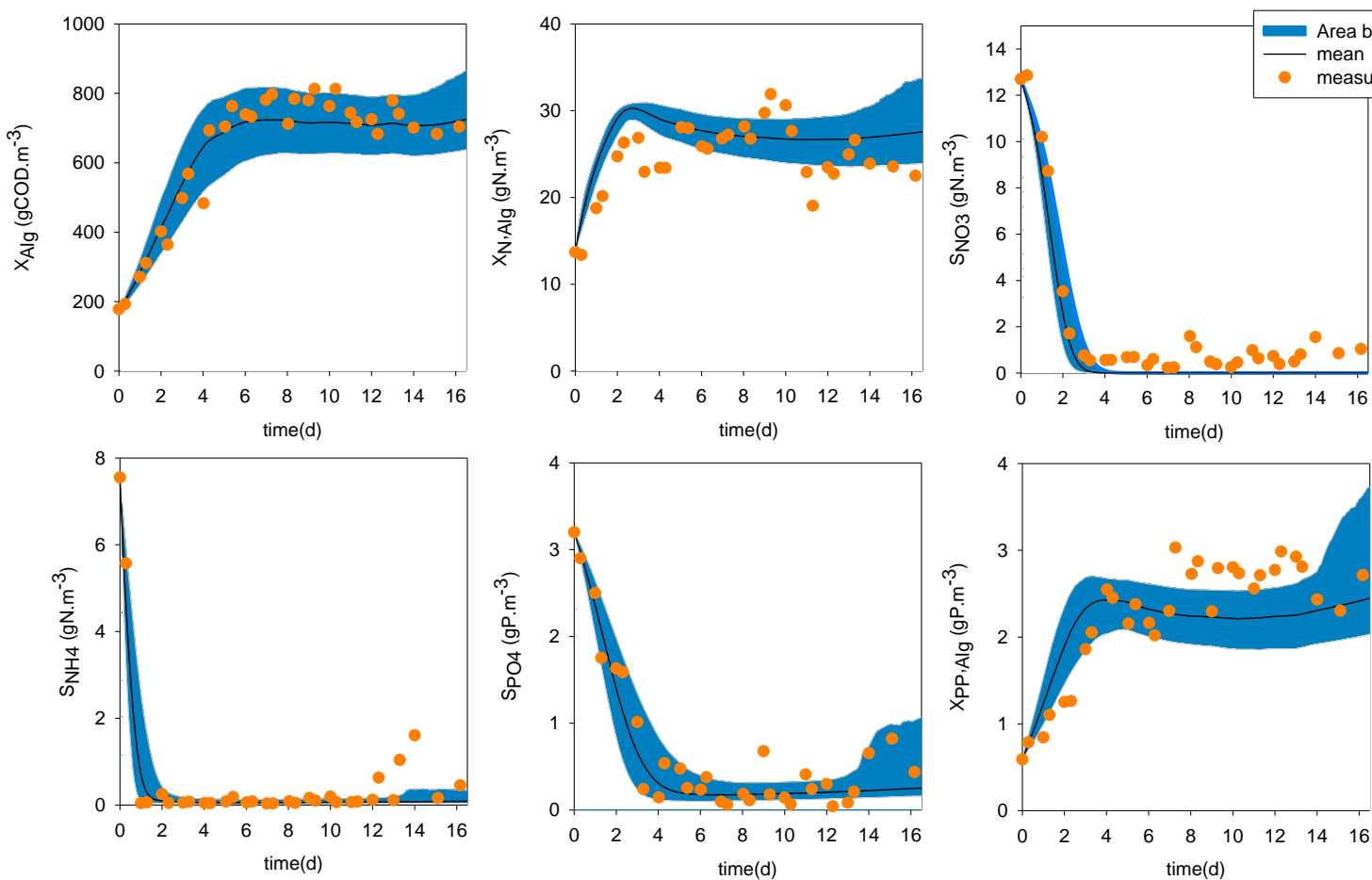

Figure S19: Simulation using model CL- 3 (one-dimensional layer model) with the mean values of the parameters estimated. The uncertainty bands are shown in blue. The TSS is used to calculate the attenuation coefficient $\left(\mathrm{k}_{\mathrm{a}}\right)$. 


\section{$\underline{\text { References }}$}

Akaike, H., 1973. Information theory and an extension of the maximum likelihood principle, in: International Symposium on Information Theory. pp. 267-281.

Dotto, C.B.S., Mannina, G., Kleidorfer, M., Vezzaro, L., Henrichs, M., McCarthy, D.T., Freni, G., Rauch, W., Deletic, A., 2012. Comparison of different uncertainty techniques in urban stormwater quantity and quality modelling. Water Research 46, 2545-2558.

Ramin, P., Valverde-Pérez, B., Polesel, F., Locatelli, L., Plósz, B.G., 2017. A systematic model identifcation method for chemical transformation pathways - the case of heroin biomarkers in wastewater. Scientific Reports 7, 9390.

Sin, G., Gernaey, K. V., Neumann, M.B., van Loosdrecht, M.C.M., Gujer, W., 2009. Uncertainty analysis in WWTP model applications: A critical discussion using an example from design. Water Research 43, 2894-2906.

Wágner, D.S., Valverde-Pérez, B., Sæbø, M., de la Sotilla, M.B., Van Wagenen, J., Smets, B.F., Plósz, B.G., 2016. Towards a consensus-based biokinetic model for green microalgae - the ASMA. Water Research 103, 485-499. 